\title{
CAT(-1)-SPACES, DIVERGENCE GROUPS AND THEIR COMMENSURATORS
}

\author{
M. BURGER AND S. MOZES
}

\section{INTRODUCTION}

A CAT(-1)-space is a metric geodesic space in which every geodesic triangle is thinner than its associated comparison triangle in the hyperbolic plane ([B], [Bri$\mathrm{Ha}],[\mathrm{Gr}])$. The CAT(-1)-property is one among many possible generalizations to singular spaces of the notion of negative curvature. Important examples of CAT(-1)-spaces include Riemannian manifolds of sectional curvature $k \leq-1$ and their convex subsets ([B-G-S]), metric trees and piecewise hyperbolic cell complexes ([Mou],[Da], [Hag], [Be 1],[Be 2],[B-Br]).

In this paper we establish certain superrigidity results for isometric actions of a group $\Lambda$ on a $\mathrm{CAT}(-1)$-space in the following two settings:

A. The group $\Lambda$ is a subgroup of a locally compact group $G$ with $\Gamma<\Lambda<$ $\operatorname{Com}_{G} \Gamma$, where $\Gamma<G$ is a sufficiently large discrete subgroup and $\operatorname{Com}_{G} \Gamma=\{g \in$ $G: g^{-1} \Gamma g$ and $\Gamma$ share a subgroup of finite index $\}$ is the commensurator of $\Gamma$ in $G$.

B. The group $\Lambda$ is an irreducible lattice in $G:=\prod_{\alpha=1}^{n} G_{\alpha}\left(k_{\alpha}\right)$, where each $G_{\alpha}$ is a semisimple algebraic group defined over a local field $k_{\alpha}$.

The issues addressed in this paper are motivated on one hand by earlier work of G.A. Margulis ([Ma]) dealing with the linear representation theory of $\Lambda$, where in case A, $G$ is a semisimple group and $\Gamma<G$ a lattice, and on the other hand by the results of Lubotzky, Mozes and Zimmer ([L-M-Z]) concerning isometric actions of $\Lambda$ on trees, where $\Gamma<\Lambda<\operatorname{Com}_{G} \Gamma, G$ is the group of automorphisms of a regular tree and $\Gamma<G$ is a lattice.

Our approach to establishing superrigidity results is based on ergodic theoretic methods developed by Margulis ([Ma],[Zi 3],[A'C-B]). In this context, the following notion of boundary of a locally compact group $\Gamma$ will be useful: let $B$ be a standard Borel space on which $\Gamma$ acts by Borel automorphisms preserving a $\sigma$-finite measure class $\mu$.

Definition. $(B, \mu)$ is a weak $\Gamma$-boundary if

(1) for any continuous affine action of $\Gamma$ on a separable locally convex space $E$ and any $\Gamma$-invariant compact convex subset $A \subset E$, there exists a $\mu$-measurable $\Gamma$-equivariant map $\varphi: B \rightarrow A$

(2) the diagonal $\Gamma$-action on $(B \times B, \mu \times \mu)$ is ergodic.

The pair $(B, \mu)$ is called a $\Gamma$-boundary if condition (1) is replaced by

$\left(1^{\prime}\right)$ the $\Gamma$-action on $(B, \mu)$ is amenable.

Received by the editors November 18, 1993.

1991 Mathematics Subject Classification. Primary 22D40, 20E08, 22E40. 
When $\Gamma$ is a closed subgroup of a locally compact group $G$, we say that a (weak) $\Gamma$-boundary $(B, \mu)$ is a (weak) $(G, \Gamma)$-boundary, if the $\Gamma$-action extends to a measure class preserving Borel $G$-action.

Let now $Y$ be a proper CAT $(-1)$-space, that is, a CAT $(-1)$-space in which all closed balls are compact. In particular, its group of isometries $\operatorname{Is}(Y)$ is locally compact, second countable and $Y$ admits an $\mathrm{Is}(Y)$-equivariant compactification $\bar{Y}:=Y \sqcup Y(\infty)$, where $Y(\infty)$ is the visual boundary of $Y$. A subgroup $\Lambda<\operatorname{Is}(Y)$ acts $c$-minimally on $Y$ if $Y$ is the only nonempty $\Lambda$-invariant closed convex subset of $Y$ and a subgroup $H<\operatorname{Is}(Y)$ is called elementary if it admits an invariant subset $\Delta \subset \bar{Y}$ consisting of one or two points.

Theorem 0.1. Let $\Gamma$ be a discrete subgroup of a locally compact second countable group $G$ and $\Lambda<G$ with $\Gamma<\Lambda<\operatorname{Com}_{G} \Gamma$. We assume that there exists a pair $(B, \mu)$ which is a weak $\left(G, \Gamma^{\prime}\right)$-boundary for any subgroup of finite index $\Gamma^{\prime}<\Gamma$. Let $Y$ be a proper $C A T(-1)$-space and $\pi: \Lambda \rightarrow I s(Y)$ a homomorphism such that $\pi(\Lambda)$ acts c-minimally on $Y$ and $\pi(\Gamma)$ is not elementary. Then $\pi$ extends to a continuous homomorphism

$$
\pi_{\text {ext }}: \bar{\Lambda} \rightarrow \operatorname{Is}(Y)
$$

While it is true that the Poisson boundary of an "étalée" measure on a locally compact group $\Gamma$ is an amenable ergodic $\Gamma$-space (see [Zi 2]) we do not know whether $\Gamma$ always admits a weak $\Gamma$-boundary in our sense. Thus an important part of our paper is devoted to the construction of pairs $\Gamma<G$ which admit a weak boundary. This is carried out in the following setting: $\Gamma$ is a discrete subgroup of the group of isometries Is $(X)$ of a proper CAT $(-1)$-space $X, G=\overline{\operatorname{Com}_{\mathrm{Is}(X)} \Gamma}$ and we assume that $\Gamma$ is a divergence group (see 6.3). The notion of a divergence group is borrowed from Patterson-Sullivan theory of Kleinian groups ([Ni],[Pa],[Su]; see also $[\mathrm{Bou}],[\mathrm{Co}],[\mathrm{Co}-\mathrm{Pa} 1],[\mathrm{Ka}])$ which we generalize to $\mathrm{CAT}(-1)$-spaces in Sections 1 and 6 . As a consequence we obtain for every divergence group $\Gamma<\operatorname{Is}(X)$ a canonical measure class on $X(\infty)$, the Patterson-Sullivan measure class (see 6.3, definition) for which the following result holds.

Theorem 0.2. Let $X$ be a proper $C A T(-1)$-space, $\Gamma<\operatorname{Is}(X)$ a discrete divergence group and $G=\overline{\operatorname{Com}_{\mathrm{Is}(X)} \Gamma}$.

(1) The Patterson-Sullivan measure class $\mu$ on $X(\infty)$ is $G$-invariant and $(X(\infty), \mu)$ is a weak $\left(G, \Gamma^{\prime}\right)$-boundary for any subgroup of finite index $\Gamma^{\prime}<\Gamma$.

(2) Let $Y$ be a proper CAT(-1)-space and $\pi: \Gamma \rightarrow \operatorname{Is}(Y)$ a homomorphism such that $\pi(\Gamma)$ is not elementary. Then there exists a unique $\Gamma$-equivariant measurable map $\varphi: X(\infty) \rightarrow Y(\infty)$ and it takes almost all its values in the limit set $\mathcal{L}_{\pi(\Gamma)}$ of $\pi(\Gamma)$.

Remark. It follows from [Ad 2], that when $Y$ is at most of exponential growth, the action of any closed subgroup of $\operatorname{Is}(Y)$ on $Y(\infty)$ is universally amenable. In this case, Theorem 0.2 implies that $(X(\infty), \mu)$ is a $(G, \Gamma)$-boundary.

Corollary 0.3. Let $X, Y$ be proper $C A T(-1)$-spaces, $\Gamma<\operatorname{Is}(X)$ a discrete divergence group, $\Lambda<\operatorname{Is}(X)$ a subgroup such that $\Gamma<\Lambda<\operatorname{Com}_{\operatorname{Is}(X)} \Gamma$ and $\pi: \Lambda \rightarrow \operatorname{Is}(Y)$ a homomorphism such that $\pi(\Lambda)$ acts c-minimally and $\pi(\Gamma)$ is not elementary. Then $\pi$ extends to a continuous homomorphism

$$
\pi_{\text {ext }}: \bar{\Lambda} \rightarrow \operatorname{Is}(Y)
$$


As examples of situations to which the above results apply we mention

(1) $\Gamma<G$ is a lattice where $G$ is a connected semisimple Lie group without compact factors. When $\Gamma$ is irreducible, the group $\operatorname{Com}_{G} \Gamma$ is nondiscrete if and only if $\Gamma$ is arithmetic, in which case $\operatorname{Com}_{G} \Gamma$ is dense in $G$ ([Ma]).

(2) $\Gamma<$ Aut $\mathcal{T}$ is a uniform tree lattice, that is, $\mathcal{T}$ is a locally finite tree and $\Gamma \backslash \mathcal{T}$ is a finite graph. According to $[\mathrm{Li}], \mathrm{Com}_{\text {Aut }} \mathcal{T}$ is dense in Aut $\mathcal{T}$ (see 8.1).

(3) $\Gamma<$ Aut $\mathcal{T}_{4}$ is the fundamental group of the Cayley graph of the free abelian group on two generators. Then $\Gamma$ is a divergence group whose commensurator is dense in Aut $\mathcal{T}_{4}$ (see 7.5 and 8.2).

(4) $\Gamma<$ Aut $\mathcal{T}$ is the fundamental group of the graph of groups (see 7.4 and 8.3)

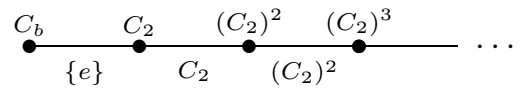

Again, $\Gamma$ is a divergence group whose commensurator is dense in Aut $\mathcal{T}$.

Next we consider actions of irreducible lattices in higher rank semisimple Lie groups on proper CAT $(-1)$-spaces. As a consequence of uniqueness results concerning certain boundary maps (see Section 3) and Margulis' factor theorem we obtain

Theorem 0.4. Let $\Gamma$ be an irreducible lattice in $G=\prod_{\alpha=1}^{n} G_{\alpha}\left(k_{\alpha}\right)$, where $G_{\alpha}$ is a simply connected, $k_{\alpha}$-almost simple, $k_{\alpha}$-isotropic group and $\sum_{\alpha=1}^{n} \operatorname{rank}_{k_{\alpha}} G_{\alpha} \geq 2$. Let $Y$ be a proper $C A T(-1)$-space and $\pi: \Gamma \rightarrow \operatorname{Is}(Y)$ a homomorphism such that $\pi(\Gamma)$ is not elementary and acts c-minimally on $Y$. Then $\pi$ extends to a continuous homomorphism $\pi_{\mathrm{ext}}: G \rightarrow \operatorname{Is}(Y)$ which factors via a proper homomorphism of some $k_{\alpha}$-rank one factor of $G$.

Corollary 0.5. Let $\Gamma$ be an irreducible lattice in $G=\prod_{\alpha=1}^{n} G_{\alpha}\left(k_{\alpha}\right)$, where $G_{\alpha}$ is a simply connected, $k_{\alpha}$-almost simple, $k_{\alpha}$-isotropic group and $\operatorname{rank}_{k_{\alpha}} G_{\alpha} \geq 2$ for every $1 \leq \alpha \leq n$. Let $Y$ be a proper $C A T(-1)$-space such that $\operatorname{Is}(Y)$ has finite critical exponent, and $\pi: \Gamma \rightarrow \operatorname{Is}(Y)$ a homomorphism. Then there exists a $\pi(\Gamma)$-fixed point in $Y$.

Remark. When $G=\mathbb{G}(\mathbb{R})(n=1)$ and $Y$ is a proper geodesic hyperbolic metric space with at most exponential growth, Corollary 0.5 is due to S. Adams ([Ad 2], Theorem 11.2).

This paper is organized as follows.

In Sections 1 and 6 we develop the Patterson-Sullivan theory for a closed subgroup $G<\operatorname{Is}(X)$, where $X$ is a proper $\operatorname{CAT}(-1)$-space, and prove Theorem 0.2 in Section 6. In Section 2 we construct an $\operatorname{Is}(X)$-equivariant center of mass map, which associates a point in $X$ to every positive measure $\mu$ on $X(\infty)$ whose support contains at least three points. In Section 3 we establish uniqueness results for $\Gamma$-equivariant measurable maps defined on a weak $\Gamma$-boundary with values in the space of compact subsets of $X(\infty)$. In Section 4 we prove Theorem 0.1, while in Section 5 we prove Theorem 0.4 and Corollary 0.5. Sections 7 and 8 are devoted to the construction of divergence groups $\Gamma<\operatorname{Is}(X)$ with dense commensurator, where $X$ is a locally finite tree.

\section{Patterson's construction, amenability}

1.1. Let $X$ be a proper metric space, i.e. closed balls are compact. The group Is $(X)$ of isometries of $X$ endowed with compact-open topology is locally compact 
and second countable. It acts on $X$ with closed orbits and compact stabilizers. Moreover, Is $(X) \backslash X$ endowed with quotient distance is again proper.

The horofunction compactification $\bar{X}$ of $X$ is the closure of the image of the injective map

$$
\begin{aligned}
X & \longrightarrow C(X) / \sim \\
x & \longmapsto\left[d_{x}\right],
\end{aligned}
$$

where $C(X)$ is the space of continuous functions on $X$ with topology of uniform convergence on compactas, $C(X) / \sim$ is its quotient by the subspace of constant functions and $d_{x}: X \rightarrow \mathbb{R}$ is the distance function to $x \in X$ (see [B-G-S], $\S 3.1$ ).

The action of $\operatorname{Is}(X)$ on $X$ extends to a continuous action on $\bar{X}$ by homeomorphisms. For $\xi \in X(\infty):=\bar{X} \backslash X$, any representative $\beta_{\xi} \in C(X)$ is called a horofunction based at $\xi$. Observe that $B_{\xi}(x, y)=\beta_{\xi}(x)-\beta_{\xi}(y)$ is independent of the chosen representative $\beta_{\xi}$. Alternatively, $(\xi, x, y) \mapsto B_{\xi}(x, y)$ is the continuous extension to $X(\infty) \times X \times X$ of the function $(z, x, y) \longmapsto B_{z}(x, y)=d(x, z)-d(y, z)$.

1.2. Definitions. For a positive Radon measure $m$ on $X$, the number

$$
\delta:=\inf \left\{s \in[0, \infty]: \int_{X} e^{-s d(x, y)} d m(y)<+\infty\right\}
$$

is independent of $x \in X$ and we call it the critical exponent of $m$. Observe that $\delta$ may be infinite. The critical exponent $\delta_{G}$ of a closed subgroup $G<\operatorname{Is}(X)$ is by definition the critical exponent of a positive $G$-invariant measure supported on a $G$-orbit in $X$. Equivalently:

$$
\delta_{G}:=\inf \left\{s \in[0, \infty]: \int_{G} e^{-s d(x, g y)} d g<+\infty\right\} .
$$

An $\alpha$-dimensional density for a closed subgroup $G<\operatorname{Is}(X)$ is a continuous $G$ equivariant map

$$
\begin{aligned}
\mu: X & \longrightarrow M^{+}(X(\infty)) \\
x & \longmapsto \mu_{x}
\end{aligned}
$$

such that

$$
\frac{d \mu_{x}(\xi)}{d \mu_{y}(\xi)}=e^{-\alpha B_{\xi}(x, y)} \quad \forall x, y \in X
$$

1.3. A straightforward generalization of a construction due to Patterson (see $[\mathrm{Pa}]$ ) leads to the result that any closed $\operatorname{subgroup} G<\operatorname{Is}(X)$ of finite critical exponent $\delta$ has a $\delta$-dimensional density. We now turn to the main points of the construction.

Proposition 1.1. Let $m$ be a positive Radon measure on $X, \delta$ its critical exponent, and $G:=\left\{g \in \operatorname{Is}(X): g_{*} m=m\right\}$. Assume $m(X)=+\infty$ and $\delta<+\infty$. Then there exists a $\delta$-dimensional density $x \rightarrow \mu_{x}$ for $G$ such that

$$
\operatorname{supp} \mu_{x} \subset \overline{\operatorname{supp}} m \cap X(\infty), \forall x \in X \text {. }
$$


Lemma $1.2([\mathrm{~Pa}])$. Let $\mu$ be a positive Radon measure on $[0, \infty)$ and assume that its critical exponent $\delta$ is finite and positive. Then there exists a continuous increasing function $h: \mathbb{R}_{+} \rightarrow \mathbb{R}_{+}$such that

(a) the integral $\int e^{-s t} h\left(e^{t}\right) d \mu(t)$ converges for $s>\delta$ and diverges at $s=\delta$;

(b) $\forall \epsilon>0, \exists t_{0}$ such that $\forall t \geq t_{0}$ and $s>1 h(s t) \leq t^{\epsilon} h(s)$.

The proof given in $[\mathrm{Pa}]$ for $\mu$ purely atomic goes over verbatim to the general case.

Given a measure $m$ on $X$ satisfying the hypothesis of Proposition 1.1, we define for $s>\delta, x, x_{0} \in X, \zeta \in \bar{X}$ :

$$
d \mu_{s, x}(\zeta):=\frac{e^{-s d(x, \zeta)} h\left(e^{d(x, \zeta)}\right) \cdot d m(\zeta)}{\int_{X} e^{-s d\left(x_{0}, y\right)} h\left(e^{d\left(x_{0}, y\right)}\right) d m(y)},
$$

where $h \equiv 1$ if $\delta=0$ and, if $\delta>0, h$ is the function associated by Lemma 1.2 to the direct image of $m$ via the proper map

$$
\begin{array}{rlr}
X & \longrightarrow & {[0, \infty)} \\
\zeta & \longmapsto d\left(x_{0}, \zeta\right) .
\end{array}
$$

Using the properties of $h$, one verifies that the family of $G$-equivariant continuous maps

$$
\begin{array}{ccc}
X & \longrightarrow & M^{+}(\bar{X}) \\
x & \longmapsto & \mu_{s, x}
\end{array}, \quad \delta<s \leq \delta+1,
$$

is equicontinuous and uniformly bounded on compact sets. This family is therefore relatively compact in the space of continuous maps $C\left(X, M^{+}(X)\right)$ endowed with the topology of uniform convergence on compact sets. It follows from Lemma 1.2(a) that any accumulation point $x \rightarrow \mu_{x}$ of this family takes its values in $M^{+}(X(\infty))$.

We have for $x, y \in X$ and $s>\delta$ :

$$
\frac{d \mu_{s, x}(\zeta)}{d \mu_{s, y}(\zeta)}=e^{-s B_{\zeta}(x, y)} \cdot \frac{h(d(x, \zeta))}{h(d(y, \zeta))}
$$

The function $\zeta \rightarrow \frac{h(d(x, \zeta))}{h(d(y, \zeta))}$ extends continuously to $\bar{X}$ and its value on $X(\infty)$ is 1 (Lemma 1.2(b)). The family of functions

$$
\begin{aligned}
& \bar{X} \longrightarrow \mathbb{R} \\
& \zeta \longmapsto e^{-s B_{\zeta}(x, y)}, \quad \delta \leq s \leq \delta+1,
\end{aligned}
$$

is compact. It follows then from $(* *)$ that any accumulation point $x \mapsto \mu_{x}$ of the family $\left\{x \mapsto \mu_{s, x}: \delta<s \leq \delta+1\right\}$ satisfies $\frac{d \mu_{x}(\xi)}{d \mu_{y}(\xi)}=e^{-\delta B_{\xi}(x, y)}$ and is therefore a $\delta$-dimensional density for $G$.

1.4. Let $G<\operatorname{Is}(X)$ be a closed subgroup with finite critical exponent $\delta$. Fix a left Haar measure $d g$ on $G$, let $m$ be its direct image via the orbital map $G \rightarrow X$, $g \rightarrow g \cdot x_{0}$, and $x \rightarrow \mu_{s, x}, s>\delta$, the corresponding family of maps defined in 1.3 $(*)$. Let $x \rightarrow \mu_{x}$ be some limit density of the above family and $\mu$ its measure class. Then we have 
Proposition 1.3. For any compact metric space $P$ on which $G$ acts continuously, there exists a $\mu$-measurable $G$-equivariant map

$$
\varphi: X(\infty) \rightarrow M^{1}(P) .
$$

Proof. The diagonal action of $G$ on $\bar{X} \times P$ is continuous and the $G$-orbits in $X \times P$ are closed with compact stabilizers. Fix $p_{0} \in P$ and let $\widetilde{m}$ be the direct image of $d g$ via the orbital map $G \rightarrow X \times P, g \mapsto g\left(x_{0}, p_{0}\right)$. For $s>\delta, x \in X, \zeta \in \bar{X}, p \in P$, define:

$$
d \widetilde{\mu}_{s, x}(\zeta, p)=\frac{e^{-s d(x, \zeta)} h\left(e^{d(x, \zeta)}\right) d \widetilde{m}(\zeta, p)}{\int_{X} e^{-s d\left(x_{0}, y\right)} h\left(e^{d\left(x_{0}, y\right)}\right) d m(y)} .
$$

Let $\pi_{*}: M^{+}(\bar{X} \times P) \rightarrow M^{+}(\bar{X})$ be the continuous $G$-equivariant map induced by the projection $\pi: \bar{X} \times P \rightarrow \bar{X}$. Observe that $\pi_{*}\left(\widetilde{\mu}_{s, x}\right)=\mu_{s, x}$. We may assume that for some sequence $s_{n} \rightarrow \delta$, the sequence $\left\{x \mapsto \mu_{s_{n}, x}: n \in \mathbb{N}\right\}$ converges to $x \mapsto \mu_{x}$ and that $\left\{x \mapsto \widetilde{\mu}_{s_{n}, x}: n \in \mathbb{N}\right\}$ converges to a continuous $G$-equivariant map

$$
\begin{aligned}
X & \longrightarrow M^{+}(X(\infty) \times P) \\
x & \longmapsto \widetilde{\mu}_{x}
\end{aligned}
$$

with $\pi_{*}\left(\widetilde{\mu}_{x}\right)=\mu_{x}, \forall x \in X$. One verifies, as in the proof of Proposition 1.1, that

$$
\frac{d \widetilde{\mu}_{x}(\xi, p)}{d \widetilde{\mu}_{y}(\xi, p)}=e^{-\delta B_{\xi}(x, y)} \quad \forall x, y \in X,(\xi, p) \in X(\infty) \times P .
$$

In particular,

$$
\frac{d\left(g_{*} \widetilde{\mu}_{x}\right)(\xi, p)}{d \widetilde{\mu}_{x}(\xi, p)}=e^{-\delta B_{\xi}(g x, x)} \quad \forall g \in G .
$$

Fix $x \in X$. Since $\pi_{*}\left(\widetilde{\mu}_{x}\right)=\mu_{x}$, there is a measurable map $\varphi: X(\infty) \rightarrow M^{1}(P)$ such that for all $\psi \in C(X(\infty) \times P)$ :

$$
\int \psi(\xi, p) d \widetilde{\mu}_{x}(\xi, p)=\int_{X(\infty)} d \mu_{x}(\xi) \int_{P} \psi(\xi, p) d \varphi(\xi)(p) .
$$

For all $g \in G$ we have

$$
\begin{aligned}
\int_{X(\infty)} d \mu_{x}(\xi) e^{-\delta B_{\xi}(g x, x)} \int_{P} \psi(\xi, p) d \varphi(\xi)(p) \\
=\int \psi(\xi, p) e^{-\delta B_{\xi}(g x, x)} d \widetilde{\mu}_{x}(\xi, p)=\int \psi(g \xi, g p) d \widetilde{\mu}_{x}(\xi, p) \\
=\int_{X(\infty)} d \mu_{x}(\xi) \int_{P} \psi(g \xi, g p) d \varphi(\xi)(p) \\
=\int_{X(\infty)} d \mu_{x}(\xi) e^{-\delta B_{\xi}(g x, x)} \int_{P} \psi(\xi, g p) d \varphi\left(g^{-1} \xi\right)(p) .
\end{aligned}
$$

Applying this to $\psi(\xi, p)=\psi_{1}(\xi) \psi_{2}(p)$, we get

$$
\int_{P} \psi_{2}(p) d \varphi(\xi)(p)=\int_{P} \psi_{2}(g p) d \varphi\left(g^{-1} \xi\right)(p)
$$

and hence $\varphi$ is $G$-equivariant.

Q.E.D.

As an immediate consequence of Proposition 1.3, we have 
Corollary 1.4. For any continuous affine action of $G$ on a locally convex separable space $E$ and any compact convex $G$-invariant subset $A \subset E$, there exists a $\mu$ measurable G-equivariant map $\varphi: X(\infty) \rightarrow A$.

We refer to [Ad 1], Theorem 5.1, for related results on the amenability of the action of a hyperbolic group on its boundary.

Lemma 1.5. Let $X$ be a proper metric space and $G<\operatorname{Is}(X)$ a closed subgroup with finite critical exponent $\delta_{G}$. Then, for any closed subgroup $H<G$ we have $\delta_{H} \leq \delta_{G}$.

Proof. Let $d g$, resp. $d h$, be left invariant Haar measures on $G$, resp. $H$, and $q: G \rightarrow(0, \infty)$ a continuous function such that $q(g h)=q(g) \Delta_{G}(h) \Delta_{H}(h)^{-1}$, $g \in G, h \in H$, where $\Delta_{G}$, resp. $\Delta_{H}$, are the modular functions of $G$, resp. $H$. Then (see [Re]) there is a $G$-quasi-invariant positive Radon measure $\mu$ on $G / H$ such that

$$
\int_{G} f(g) d g=\int_{G / H} d \mu(\dot{g}) \int_{H} q(g h) f(g h) d h
$$

for any positive measurable function $f$. Using this formula one verifies for $s>\delta_{G}$ :

$$
\int_{G} e^{-s d(g x, x)} d g=\int_{G / H} d \mu(\dot{g}) q(g) \Delta_{G}\left(g^{-1}\right) \int_{H} e^{-s d\left(h g^{-1} x, x\right)} d h .
$$

In particular, $\int_{H} e^{-s d\left(h g^{-1} x, x\right)} d h$ is finite for some $g$, hence $s \geq \delta_{H}$.

Q.E.D.

Proposition 1.6. Let $X$ be a proper $C A T(-1)$-space and $G<\operatorname{Is}(X)$ a closed subgroup with finite critical exponent. For every $\xi \in X(\infty)$, the closed subgroup $\operatorname{Stab}_{G}(\xi)$ is amenable.

Proof. Fix a base point $p \in X$ and fix a choice of horofunctions $\beta_{\eta}, \eta \in X(\infty)$, such that $\beta_{\eta}(p)=0$. Let $H<\operatorname{Stab}_{G}(\xi)$ be the kernel of the continuous homomorphism $\chi_{\xi}: \operatorname{Stab}_{G}(\xi) \rightarrow \mathbb{R}$ defined by

$$
\beta_{\xi}(g x)-\beta_{\xi}(x)=\chi_{\xi}(g), \forall x \in X, g \in \operatorname{Stab}_{G}(\xi) .
$$

Since $\mathbb{R}$ is abelian and $H$ is closed normal, it suffices to show that $H$ is amenable. Let $m$ be an $H$-invariant measure supported on the orbit $H \cdot p \subset X$. If $m$ is finite, then, since $\operatorname{Stab}_{H}(p)$ is compact, $H$ admits a finite Haar measure and hence is compact. Assume $m(X)=+\infty$. We claim that $\overline{\operatorname{supp} m} \cap X(\infty)=\{\xi\}$. Since supp $m$ is unbounded and contained in $H \cdot p$ we have

$$
\emptyset \neq X(\infty) \cap \overline{\operatorname{supp} m} \subset X(\infty) \cap \overline{\beta_{\xi}^{-1}(0)} .
$$

For $z \in \beta_{\xi}^{-1}(0)$, a computation shows that the Gromov scalar product of $z$ and $\xi$ w.r.t. $p$ equals

$$
(z \cdot \xi)_{p}=\frac{1}{2} d(z, p) .
$$

Therefore, if $\eta \in \overline{\beta_{\xi}^{-1}(0)} \cap X(\infty)$, we have

$$
(\eta \cdot \xi)_{p}=+\infty
$$


and hence $\eta=\xi$, which proves the claim. Let $P$ be a compact metric space on which $H$ acts continuously. Since $H$ has finite critical exponent (Lemma 1.5), it follows from Propositions 1.1 and 1.3 that there exists an $H$-equivariant measurable map $\varphi:\{\xi\} \rightarrow M^{1}(P)$, and therefore an $H$-invariant probability measure on $P$. Q.E.D.

Remark. The product $X=[0, \infty) \times \mathbb{H}^{2}$ with Riemannian metric $(d t)^{2}+e^{-2 t} \frac{|d z|^{2}}{y^{2}}$, where $\mathbb{H}^{2}$ denotes the upper half-plane, is a $\operatorname{CAT}(-1)$-space on which $\operatorname{SL}(2, \mathbb{R})$ acts isometrically, fixing a point in $X(\infty)$.

Proposition 1.7. Let $X$ be a proper geodesic space such that $\operatorname{Is}(X) \backslash X$ is compact. Then $\operatorname{Is}(X)$ has finite critical exponent.

Proof. Fix $x_{0} \in X, D>0$ such that $\bigcup_{g \in G} g B\left(x_{0}, D-2\right)=X$ and $F \subset G$ finite such that $\bigcup_{g \in F} g B\left(x_{0}, D-2\right) \supset S\left(x_{0}, D\right)$.

For every $x \in X$ with $d\left(x_{0}, x\right) \geq D$ there exists $g \in F$ such that for all $h \in U_{g}:=$ $\left\{g^{\prime} \in G: d\left(g^{\prime} x_{0}, g x_{0}\right)<1\right\}, d\left(h x_{0}, x\right)<d\left(x_{0}, x\right)-1$. Indeed, the point $p \in S\left(x_{0}, D\right)$ on the geodesic segment joining $x_{0}$ to $x$ verifies $d\left(g x_{0}, p\right)<D-2$ for some $g \in F$. This implies $d\left(g x_{0}, x\right)<d\left(x_{0}, x\right)-2$ and hence $d\left(h x_{0}, x\right)<d\left(x_{0}, x\right)-1$, for all $h \in U_{g}$.

Fix $R>0$ such that $h^{-1} B\left(x_{0}, D\right) \subset B\left(x_{0}, R\right), \forall h \in U:=\bigcup_{g \in F} U_{g}$. For $f \in L^{1}(G)$ and $F \in L^{1}(X, \bar{m})$ define

$$
f * F(x):=\int_{G} f(g) F\left(g^{-1} x\right) d m(g),
$$

where $m$ denotes a Haar measure on $G$ and $\bar{m}$ the corresponding $G$-invariant measure on $G \cdot x_{0} \subset X$. For $n \in \mathbb{N}$, set

$$
f_{n}(x):=\chi_{U} * \ldots * \chi_{U} * \chi_{B\left(x_{0}, R\right)}(x)
$$

and $\alpha:=\min _{g \in F} m\left(U_{g}\right)$. We claim that

$$
f_{n}(x) \geq \alpha^{n}, \quad \forall x \in B\left(x_{0}, R+n\right) .
$$

By induction on $n$, the case $n=0$ is obvious. Now fix $n \geq 1$. For every $x \in$ $B\left(x_{0}, R+n\right) \backslash B\left(x_{0}, D\right)$ there is $g \in F$ such that $d\left(h x_{0}, x\right)<d\left(x_{0}, x\right)-1<R+n-1$ for all $h \in U_{g}$; and hence $f_{n-1}\left(h^{-1} x\right) \geq \alpha^{n-1}$. For $x \in B\left(x_{0}, D\right)$ and $h \in U$, $h^{-1} x \in B\left(x_{0}, R\right)$ and therefore $f_{n-1}\left(h^{-1} x\right) \geq \alpha^{n-1}$. For $x \in B\left(x_{0}, R+n\right)$ we have therefore

$$
f_{n}(x)=\int_{G} \chi_{u}(h) f_{n-1}\left(h^{-1} x\right) d m(x) \geq \alpha \cdot \alpha^{n-1}=\alpha^{n} .
$$

The above claim now implies

$$
\alpha^{n} \bar{m}\left(B\left(x_{0}, R+n\right)\right) \leq \int f_{n}(x) d \bar{m}(x)=m(U)^{n} \cdot \bar{m}\left(B\left(x_{0}, R\right)\right)
$$

which shows that $T \rightarrow \bar{m}\left(B\left(x_{0}, T\right)\right)$ has a finite rate of exponential growth and, hence, the critical exponent of $\operatorname{Is}(X)$ is finite.

Q.E.D. 


\section{Center OF MASS}

Let $Y$ be a proper CAT(-1)-space, $\operatorname{Is}(Y)$ its group of isometries and $M_{3}^{+}(Y(\infty))$ the space of positive measures on $Y(\infty)$ whose support contains at least three points. In this section we prove

Proposition 2.1. There is an $\operatorname{Is}(Y)$-equivariant map

$$
C m: M_{3}^{+}(Y(\infty)) \longrightarrow Y,
$$

whose restriction to any $\operatorname{Is}(Y)$-orbit in $M_{3}^{+}(Y(\infty))$ is continuous.

We begin by considering the action of $\operatorname{Is}(Y)$ on $F_{n} Y(\infty):=\{S \subset Y(\infty):|S|=$ $n\}$. The space $F_{n} Y(\infty)$ being an open subset in the space of all unordered $n$ tuples of points of $Y(\infty)$ inherits a locally compact topology for which $\operatorname{Is}(Y)$ acts continuously. For $S \in F_{n} Y(\infty)$ and $y \in Y$, define

$$
F_{S}(y):=\sum_{\substack{\xi, \nu \in S \\ \xi \neq \nu}} d(y,[\xi, \nu])
$$

where $d(y,[\xi, \nu])$ is the distance from $y$ to the geodesic $[\xi, \nu]$ connecting $\xi$ to $\nu$. For all $g \in \operatorname{Is}(Y)$ and $S \in F_{n} Y(\infty)$, we have $F_{g S}=F_{S} \circ$.

Lemma 2.2. For $n \geq 3$, the group $\operatorname{Is}(Y)$ acts on $F_{n} Y(\infty)$ with closed orbits and compact stabilizers.

Proof. We observe first that for $n \geq 3$ and $K \subset F_{n} Y(\infty)$ a compact subset, the continuous function

$$
\begin{aligned}
K \times Y & \longrightarrow \mathbb{R} \\
(S, y) & \longmapsto
\end{aligned}
$$

is proper. To show the lemma, it suffices to show that given $S \in F_{n} Y(\infty)$ and a sequence $\left(g_{n}\right)_{n \in \mathbb{N}}$ in $\operatorname{Is}(Y)$ with $\lim _{n \rightarrow \infty} g_{n} S=T$ for some $T \in F_{n} Y(\infty)$, the sequence $\left(g_{n}\right)_{n \in \mathbb{N}}$ is relatively compact. Let $\lambda=\min \left\{F_{T}(y): y \in Y\right\}, V$ a compact neighborhood of $T$ in $F_{n} Y(\infty)$ and $N$ such that $g_{n} S \in V$ for all $n \geq N$. Since $\left\{\left(g_{n} S, y\right): F_{g_{n} S}(y) \leq \lambda+1\right\}$ is relatively compact in $V \times Y$, the set $\bigcup_{n \geq N} g_{n}^{-1} F_{S}^{-1}([0, \lambda+1])$ is relatively compact and hence $\left(g_{n}\right)_{n \in \mathbb{N}}$ is relatively compact.

Q.E.D.

Lemma 2.3 (Compare with [Fu], [L-M-Z]). (a) Let $\mu, \nu$ be probability measures on $Y(\infty)$ and $\left(g_{n}\right)_{n \in \mathbb{N}}$ a nonrelatively compact sequence in $\operatorname{Is}(Y)$ such that

$$
\lim _{n \rightarrow \infty} g_{n} * \mu=\nu
$$

Then the support of $\nu$ has at most two points.

(b) $M_{3}^{+}(Y(\infty))$ is open in $M^{+}(Y(\infty))$. The action of $\operatorname{Is}(Y)$ on $M_{3}^{+}(Y(\infty))$ has closed orbits and compact stabilizers.

Proof. (a) Without loss of generality we may assume that $\left(g_{n}\right)_{n+\mathbb{N}}$ tends to infinity. Suppose that the support of $\nu$ contains more than two points. This would imply that there exist three distinct points $\xi_{i} \in Y(\infty), 1 \leq i \leq 3$, and three distinct points $\zeta_{i} \in Y(\infty)$ such that for some subsequence $\left(h_{n}\right)_{n \in \mathbb{N}}$ of $\left(g_{n}\right)_{n \in \mathbb{N}}$ we have 
$\lim _{n \rightarrow \infty} h_{n} \cdot \zeta_{i}=\xi_{i}$ for $1 \leq i \leq 3$. This however implies that the sequence $\left(h_{n}\right)_{n \in \mathbb{N}}$ is bounded (Lemma 2.2) which is a contradiction.

(b) follows from (a).

Q.E.D.

We observe now that if $C: M_{3}^{+}(Y(\infty)) \longrightarrow Y$ is any $\operatorname{Is}(Y)$-equivariant map, its restriction to any $\mathrm{Is}(Y)$-orbit is continuous. Indeed, by Lemma 2.3(b), the orbital maps

$$
\begin{aligned}
& \operatorname{Is}(Y) / \operatorname{Stab}(\nu) \longrightarrow \operatorname{Is}(Y) \cdot \nu \\
& \operatorname{Is}(Y) / \operatorname{Stab}(C(\nu)) \longrightarrow \operatorname{Is}(Y) \cdot C(\nu), \quad \nu \in M_{3}^{+}(Y(\infty)) \text {, }
\end{aligned}
$$

are homeomorphisms, $C$ is $\operatorname{Is}(Y)$-equivariant, and $\operatorname{Stab}(\nu) \subset \operatorname{Stab}(C(\nu))$.

We turn now to the construction of such a map.

A. For a bounded subset $E \subset Y$ we let $\operatorname{ct}(E)$ denote the center of the unique closed ball of minimal radius containing $E$.

B. For a finite subset $S \subset Y(\infty)$ with $|S| \geq 3$, the function $F_{S}$ is proper convex and $\operatorname{Min} F_{S}:=\left\{y \in Y: F_{S}(y)=\inf F_{S}\right\}$ is therefore nonvoid bounded convex and closed. Set $c(S):=c t\left(\operatorname{Min} F_{S}\right)$.

Given now $\mu \in M_{3}^{+}(Y(\infty))$ a purely atomic measure and $a_{1} \geq \ldots \geq a_{n} \geq \ldots$ the sequence of positive values taken by the function $\rho \longmapsto \mu(\rho)$, the set $\rho_{\mu}:=\{\rho \in$ $\left.Y(\infty): \mu(\rho) \geq a_{3}\right\} \subset Y(\infty)$ is finite and contains at least three points. We define $C m(\mu):=c\left(\rho_{\mu}\right)$.

C. To a probability measure $\mu$ on $Y(\infty)$ we associate the convex function (Comp. $[\mathrm{Zi} 2])$

$$
F_{\mu}(y):=\int_{Y(\infty)} \beta_{\rho}(y) d \mu(\rho),
$$

where $\beta: Y(\infty) \times Y \longrightarrow \mathbb{R}$ is a fixed horofunction with $\beta_{\rho}(p)=0$ for all $\rho \in Y(\infty)$, and $p$ is a fixed point in $Y$.

Lemma 2.4. Assume that $\mu(\rho)<\frac{1}{2}$ for all $\rho \in Y(\infty)$. Then $F_{\mu}$ is a proper function.

Proof. Since $F_{\mu}$ is convex, it suffices to show that for any geodesic ray $r:[0, \infty) \longrightarrow$ $Y$ starting at $p, \lim _{t \rightarrow \infty} F_{\mu}(r(t))=+\infty$. Set $\xi=r(+\infty)$ and define for $R \in \mathbb{N}$

$$
V(R):=\left\{\rho \in Y(\infty): \beta_{\rho}(r(t+s))-\beta_{\rho}(r(t)) \geq s-1, \forall t \geq R, \forall s \geq 0\right\} .
$$

For every $\rho \neq \xi$ the geodesics $[\rho, \xi]$ and $r$ are asymptotic and therefore

$$
Y(\infty) \backslash\{\xi\}=\bigcup_{R \in \mathbb{N}} V(R) .
$$

Since $\mu(\xi)<\frac{1}{2}$, there exists $R \in \mathbb{N}$ with $\mu(V(R))>\frac{1}{2}$. We have then, for $t \geq R$ and $s \geq 0$,

$$
\begin{aligned}
F_{\mu}(r(t+s))-F_{\mu}(r(t)) & =\int_{V(R)}\left[\beta_{\rho}(r(t+s))-\beta_{\rho}(r(t))\right] d \mu(\rho) \\
& \quad+\int_{Y(\infty) \backslash V(R)}\left[\beta_{\rho}(r(t+s))-\beta_{\rho}(r(t))\right] d \mu(\rho) \\
& \geq(s-1) \mu(V(R))-s \cdot \mu(Y(\infty) \backslash V(R)) \\
& \geq(2 \mu(V(R))-1) s-1,
\end{aligned}
$$


which shows that $\lim _{t \rightarrow \infty} F_{\mu}(r(t))=+\infty$.

Q.E.D.

For a measure $\mu$ satisfying the hypothesis of Lemma 2.4, the set Min $F_{\mu}=$ $\left\{y \in Y: F_{\mu}(y)=\inf F_{\mu}\right\}$ is therefore nonvoid, convex and compact. Observe that, for $g \in \operatorname{Is}(Y)$, the functions $F_{g_{*} \mu}$ and $F_{\mu} \circ g^{-1}$ differ by a constant and hence $\operatorname{Min} F_{g_{*} \mu}=g\left(\operatorname{Min} F_{\mu}\right)$.

Proof of Proposition 2.1. For $\mu \in M_{3}^{+}(Y(\infty))$, let $\mu=\mu_{A}+\mu_{D}$ be the decomposition of $\mu$ into its purely atomic part $\mu_{A}$ and nonatomic part $\mu_{D}$.

If $\mu_{D}=0$, we set $C m(\mu):=C m\left(\mu_{A}\right)$ as defined in B. If $\mu_{D} \neq 0$, the measure $\nu=\mu_{D} / \mu_{D}(Y(\infty))$ satisfies the hypothesis of Lemma 2.4 and we define

$$
C m(\mu):=\operatorname{ct}\left(\operatorname{Min} F_{\nu}\right)
$$

Q.E.D.

Corollary 2.5. Let $Y$ be a proper $C A T(-1)$-space.

(1) A subgroup $H<\operatorname{Is}(Y)$ is elementary if and only if there exists an $H$-invariant probability measure on $Y(\infty)$.

(2) A closed subgroup $H<\mathrm{Is}(Y)$ with $\delta_{H}=0$ is elementary.

(3) Assume that $\operatorname{Is}(Y)$ has finite critical exponent. A closed subgroup $H<\operatorname{Is}(Y)$ is amenable if and only if it is elementary.

Proof. (1) If $H$ is elementary, there exists $\Delta \subset \bar{Y}$, an $H$-invariant subset with $1 \leq|\Delta| \leq 2$. If $\Delta \cap Y \neq \emptyset$, then $\bar{H}$ is compact and hence fixes a probability measure on $Y(\infty)$. If $\Delta \subset Y(\infty)$, then $|\Delta|^{-1} \sum_{\eta \in \Delta} \delta_{\eta}$ is an $H$-invariant probability measure on $Y(\infty)$. Conversely, let $\mu \in M^{1}(Y(\infty))$ be an invariant probability measure. Then, either $|\operatorname{supp} \mu| \leq 2$ and $H$ is elementary or $\mu \in M_{3}^{1}(Y(\infty))$ and $H$ fixes $C m(\mu) \in Y$ (Proposition 2.1).

(2) Proposition 1.1 implies that there exists a 0-dimensional density for $H$ and hence an $H$-invariant probability measure on $Y(\infty)$; so $H$ is elementary by (1).

(3) If $H$ is amenable, it fixes a probability measure on $Y(\infty)$ and is therefore elementary. If $H$ is elementary, it contains a normal subgroup of index at most 2 which fixes a point in $\bar{Y}$. It follows then from Proposition 1.6 that $H$ is amenable. Q.E.D.

\section{BOUNDARY MAPS}

3.1. Given a proper CAT(-1)-space $X$, a subgroup $G<\operatorname{Is}(X)$ and $x \in X$, the set $\overline{G \cdot x} \cap X(\infty)$ is independent of $x \in X$; it is the limit set $\mathcal{L}_{G}$ of $G$. If $G$ does not fix a point in $X(\infty)$, any nonvoid $G$-invariant closed subset $A \subset X(\infty)$ verifies $A \supset \mathcal{L}_{G}$ and therefore $G$ acts minimally on $\mathcal{L}_{G}$ ([Gh-H], 8. 3 , Proposition 25). In particular, for any closed nonvoid convex $G$-invariant subset $C \subset X$, one has $C(\infty) \supset \mathcal{L}_{G}$ and the $G$-action on $C o\left(\mathcal{L}_{G}\right) \subset X$, the closed convex hull of $\mathcal{L}_{G}$, is therefore $c$-minimal. One has the inclusion $\mathcal{L}_{G} \subset C o\left(\mathcal{L}_{G}\right)(\infty)$; we do not know if equality holds in general (see however [Bow]).

Let now $\Gamma$ be a countable group acting by Borel automorphisms on a standard Borel space $B$, preserving a $\sigma$-finite measure class $\mu$, and let $\pi: \Gamma \rightarrow \operatorname{Is}(X)$ be a homomorphism. Denote by $K(X(\infty))$ the space of closed subsets of $X(\infty)$ endowed with the Hausdorff topology. 
Proposition 3.1. Assume that the diagonal $\Gamma$-action on $(B \times B, \mu \times \mu)$ is ergodic and that $\pi(\Gamma)$ does not fix a point in $\bar{X}$. Let $F: B \rightarrow K(X(\infty))$ be a $\Gamma$-equivariant measurable map. Then, either $F(b) \supset \mathcal{L}_{\pi(\Gamma)}$ for a.e. $b \in B$, or $|F(b)|=1$ for a.e. $b \in B$.

Corollary 3.2. Assume that the diagonal $\Gamma$-action on $(B \times B, \mu \times \mu)$ is ergodic and that $\pi(\Gamma)$ is nonelementary. Then any $\Gamma$-equivariant measurable map

$$
\Phi: B \longrightarrow M^{1}(X(\infty))
$$

takes almost all its values in the subset of Dirac measures. In particular, there exists at most one $\Gamma$-equivariant measurable map

$$
\varphi: B \longrightarrow X(\infty)
$$

3.2. We begin with the following observation: for $\xi_{1}, \xi_{2}, \xi_{3}, \xi_{4} \in X(\infty)$ with $\xi_{i} \neq$ $\xi_{i+1}, 1 \leq i \leq 4$, where $\xi_{5}=\xi_{1}$, the quantity $\left[\xi_{1}, \xi_{2}, \xi_{3}, \xi_{4}\right]:=\left(\xi_{1} \cdot \xi_{2}\right)_{x}-$ $\left(\xi_{2} \cdot \xi_{3}\right)_{x}+\left(\xi_{3} \cdot \xi_{4}\right)_{x}-\left(\xi_{4} \cdot \xi_{1}\right)_{x}$ is well defined and independent of $x \in X$ and hence one has $\left[g \xi_{1}, g \xi_{2}, g \xi_{3}, g \xi_{4}\right]=\left[\xi_{1}, \xi_{2}, \xi_{3}, \xi_{4}\right], \forall g \in \operatorname{Is}(X)$. Given two closed subsets $F_{1}, F_{2} \subset X(\infty)$, containing each at least two points, the quantity

$$
h\left(F_{1}, F_{2}\right):=\min \left\{\left|\left[\xi_{1}, \xi_{2}, \xi_{3}, \xi_{4}\right]\right|: \xi_{1}, \xi_{2} \in F_{1}, \xi_{3}, \xi_{4} \in F_{2}, \xi_{i} \neq \xi_{i+1}\right\}
$$

is finite and $h\left(g F_{1}, g F_{2}\right)=h\left(F_{1}, F_{2}\right) \forall g \in \operatorname{Is}(X)$.

Proof of Proposition 3.1. Assume that there is $\xi \in X(\infty)$ such that $B_{\xi}:=\{b \in B$ : $F(b) \not \nexists \xi\}$ has measure 0 . Since $\Gamma$ is countable, we can find a $\Gamma$-invariant subset $E \subset B$ of full measure such that $F(b) \ni \xi$ for all $b \in E$. The closed $\pi(\Gamma)$-invariant subset $\bigcap_{b \in E} F(b) \subset X(\infty)$ is then nonvoid and hence contains $\mathcal{L}_{\pi(\Gamma)}$ (see 3.1).

Assume now that $B_{\xi}$ has positive measure for every $\xi \in X(\infty)$ and $|F(b)| \geq 2$ for a.e. $b \in B$. We wish to obtain a contradiction. Let $r$ be the essential value of the $\Gamma$-invariant measurable function $B \times B \rightarrow \mathbb{R},\left(b, b^{\prime}\right) \rightarrow h\left(F(b), F\left(b^{\prime}\right)\right)$.

Since $\pi(\Gamma)$ does not fix a point in $\bar{X}$, the limit set $\mathcal{L}_{\pi(\Gamma)}$ contains at least two points; in particular there exists $\gamma \in \Gamma$ such that $\pi(\gamma)$ is hyperbolic (see [Gr], 8.1.A). Let $\xi_{+}$, resp. $\xi_{-}$, denote the attractive, resp. repelling, fixed point of $\pi(\gamma)$ in $X(\infty)$. We claim that for every $\left(b, b^{\prime}\right) \in B_{\xi_{+}} \times B_{\xi_{-}}, \lim _{n \rightarrow \infty} h\left(F(b), \pi(\gamma)^{n} F\left(b^{\prime}\right)\right)=+\infty$.

Indeed, fix $x \in X$; since $\xi_{+} \notin F(b)$ there is a neighborhood $V_{0}$ of $\xi_{+}$such that

$$
\ell:=\sup \left\{\left(\xi_{1} \cdot \xi_{2}\right)_{x}: \xi_{1} \in V_{0}, \xi_{2} \in F(b)\right\}<+\infty .
$$

For $n \in \mathbb{N}$, let $V_{n} \subset V_{0}$ be a neighborhood of $\xi_{+}$such that $\left(\xi_{1} \cdot \xi_{2}\right)_{x} \geq n$ for all $\xi_{1}, \xi_{2} \in V_{n}$. Since $F\left(b^{\prime}\right) \not \supset \xi_{-}$, there exists $k_{0} \geq 0$ such that for all $k \geq k_{0}$, $\pi(\gamma)^{k} F\left(b^{\prime}\right) \subset V_{n}$. The definition of $h$ implies then $h\left(F(b), \pi(\gamma)^{k} F\left(b^{\prime}\right)\right) \geq n-2 \ell$, which proves the claim.

For $S:=\left\{\left(b, b^{\prime}\right) \in B \times B: h\left(F(b), F\left(b^{\prime}\right)\right) \geq r+1\right\}$, the claim implies

$$
\bigcup_{n \geq 0}\left(e, \gamma^{-n}\right) S \supset B_{\xi_{+}} \times B_{\xi_{-}} .
$$

Since $B_{\xi_{+}} \times B_{\xi_{-}}$has positive measure, $S$ must have positive measure, contradicting the definition of $r$. Therefore $|F(b)|=1$ for a.e. $b \in B$.

Q.E.D. 
Proof of Corollary 3.2. Composing $\Phi$ with the Borel map supp: $M^{1}(X(\infty)) \rightarrow$ $K(X(\infty))$, which to every probability measure associates its support (see [A'C-B], section 4), we obtain a $\Gamma$-equivariant measurable map $F: B \rightarrow K(X(\infty))$. If $|F(b)|>1$ for a.e. $b \in B$, then $F(b) \supset \mathcal{L}_{\pi(\Gamma)}$ for a.e. $b \in B$ (Proposition 3.1). Since $\pi(\Gamma)$ is nonelementary, $\left|\mathcal{L}_{\pi(\Gamma)}\right| \geq 3$ and hence $\Phi$ takes almost all its values in $M_{3}^{1}(X(\infty))$. Composing $\Phi$ with the center of mass map (see Lemma 2.3 (b)) and Proposition 2.1), we obtain a $\Gamma$-equivariant measurable map $f: B \rightarrow X$. Let $d$ be the essential value of the $\Gamma$-invariant measurable function $\left(b, b^{\prime}\right) \rightarrow d\left(f(b), f\left(b^{\prime}\right)\right)$. There exist $b \in B$ and a $\Gamma$-invariant subset $E \subset B$ of full measure, such that $d\left(f(b), f\left(b^{\prime}\right)\right)=d, \forall b^{\prime} \in E$. Hence $\left\{f\left(b^{\prime}\right): b^{\prime} \in E\right\}$ is a $\pi(\Gamma)$-invariant bounded subset of $X$, which contradicts the assumption that $\pi(\Gamma)$ is nonelementary. This shows $|F(b)|=1$ for a.e. $b \in B$ and hence $\Phi$ takes almost all its values in the subset of Dirac measures. If $\varphi_{1}, \varphi_{2}: B \rightarrow X(\infty)$ are $\Gamma$-equivariant measurable maps, $\Phi(b):=\frac{1}{2} \delta_{\varphi_{1}(b)}+\frac{1}{2} \delta_{\varphi_{2}(b)}$ must be a Dirac measure for a.e. $b \in B$, hence $\varphi_{1}=\varphi_{2}$ a.e.

Q.E.D.

\section{SUPERRIGIDITY FOR COMMENSURATORS}

In this section we prove Theorem 0.1. The main ingredients in its proof are the uniqueness results (Corollary 3.2) of $\S 3$ and the general Proposition 4.1 below. This proposition will also be used in the proof of Theorem 0.4.

Proposition 4.1. Let $G$ be a locally compact, second countable group, $B$ a standard Borel space, and $G \times B \rightarrow B$ a Borel action preserving a $\sigma$-finite measure class $\mu$. Let $\Lambda<G$ be a dense subgroup, $Y$ a proper $C A T(-1)$-space and $\pi: \Lambda \rightarrow \operatorname{Is}(Y)$ a homomorphism such that $\pi(\Lambda)$ is nonelementary and acts c-minimally on $Y$. Assume that there exists a $\Lambda$-equivariant measurable map $\varphi: B \rightarrow Y(\infty)$. Then $\pi$ extends continuously to $G$.

Remark 4.2. We begin with the following general observation which will again be needed in the proof of Theorem 0.4. Let $(S, m)$ be a $\sigma$-finite measure space and $F\left(S, M^{1}(Y(\infty))\right)$ the space of measurable maps from $S$ to $M^{1}(Y(\infty))$, endowed with the topology of convergence in measure. This space is metrizable complete, and from the $\operatorname{Is}(Y)$-action on $M^{1}(Y(\infty))$, one obtains a continuous $\operatorname{Is}(Y)$-action on $F\left(S, M^{1}(Y(\infty))\right.$. Clearly, the set

$$
F_{3}:=\left\{f \in F\left(S, M^{1}(Y(\infty))\right):\left\{s \in S: f(s) \in M_{3}^{1}(Y(\infty))\right\} \text { has positive measure }\right\}
$$

is an open subset of $F\left(S, M^{1}(Y(\infty))\right)$. Moreover, since the $\operatorname{Is}(Y)$-orbits in $M_{3}^{1}(Y(\infty))$ are closed and have compact stabilizers, the same holds for the $\operatorname{Is}(Y)$ orbits in $F_{3}$. In particular, for every $f \in F_{3}$, the orbital map induces a homeomorphism

$$
\operatorname{Is}(Y) / \operatorname{Stab}(f) \longrightarrow \operatorname{Is}(Y)_{*} f \subset F_{3} .
$$

Proof of Proposition 4.1. Observe first that it is sufficient to prove the proposition in the case where $\Lambda$ is countable. Let now $\alpha$ be a probability measure in the class of $\mu$. Since supp $\varphi_{*}\left(g_{*} \alpha\right)=\operatorname{supp} \varphi_{*} \alpha$ for all $g \in G$ and since $\pi(\Lambda)$ is nonelementary, we have $\varphi_{*}\left(g_{*} \alpha\right) \in M_{3}^{1}(Y(\infty))$ for all $g \in G$. The map

$$
\Phi: G \longrightarrow F\left(G, M_{3}^{1}(Y(\infty))\right)
$$

defined by $\Phi(g)\left(g^{\prime}\right):=\varphi_{*}\left(g g_{*}^{\prime} \alpha\right), g, g^{\prime} \in G$, is measurable and $\Lambda$-equivariant. It follows then from Remark 4.2 and the fact that $\Lambda$ acts ergodically on $G$, that the 
essential image of $\Phi$ is contained in one Is $(Y)$-orbit. Hence, there exists $g_{0}$ such that for almost all $g \in G: \Phi\left(g_{0} g\right) \in \operatorname{Is}(Y)_{*} \Phi\left(g_{0}\right)$. Observe that the stabilizer of $\Phi\left(g_{0}\right)$ in $\operatorname{Is}(Y)$ is trivial. Indeed, for $h \in \operatorname{Is}(Y)$ with $h \Phi\left(g_{0}\right)=\Phi\left(g_{0}\right)$, we have: $h \varphi_{*}\left(g_{0} g_{*} \alpha\right)=\varphi_{*}\left(g_{0} g_{*} \alpha\right)$ for a.e. $g \in G$. In particular there exists $g_{1} \in G$ such that for all $\lambda \in \Lambda, h \pi(\lambda)_{*} \varphi\left(g_{1 *} \alpha\right)=\pi(\lambda)_{*} \varphi\left(g_{1 *} \alpha\right)$. Hence $h$ fixes pointwise the $\pi(\Lambda)$ orbit in $Y$ of $C m\left(\varphi_{*}\left(g_{1 *} \alpha\right)\right)$ and its closed convex hull; since $\pi(\Lambda)$ acts $c$-minimally, the latter coincides with $Y$. Hence $h=e$.

We obtain in this way a well-defined measurable map $\tau: G \rightarrow \operatorname{Is}(Y)$, such that for a.e. $g \in G: \Phi\left(g_{0} g\right)=\tau(g) \Phi\left(g_{0}\right)$. Using Fubini's theorem one shows that $\tau$ is a measurable homomorphism and therefore coincides a.e. with a continuous homomorphism $\tilde{\tau}: G \rightarrow \operatorname{Is}(Y)$. The $\Lambda$-equivariance of $\Phi$ implies then that $g \rightarrow$ $\tilde{\tau}\left(g_{0}^{-1} g g_{0}\right)$ gives the continuous extension of $\pi$ to $G$.

Proof of Theorem 0.1. Since $(B, \mu)$ is a weak $(G, \Gamma)$-boundary, there exists a $\Gamma$ equivariant measurable map $\varphi: B \rightarrow M^{1}(Y(\infty))$ which takes almost all its values in the subset of Dirac measures (Corollary 3.2), thus providing a $\Gamma$-equivariant measurable map $\varphi: B \rightarrow Y(\infty)$.

For $\lambda \in \Lambda$, the maps $\varphi$ and $\pi(\lambda)^{-1} \circ \varphi \circ \lambda$ are both equivariant w.r.t. the subgroup $\lambda^{-1} \Gamma \lambda \cap \Gamma$ which is of finite index in $\Gamma$. The assumption that any subgroup of finite index in $\Gamma$ acts ergodically on $B \times B$ and Corollary 3.2 imply then that $\varphi(b)=$ $\pi(\lambda)^{-1} \varphi(\lambda b)$ for a.e. $b \in B$ and all $\lambda \in \Lambda$. It follows then from Proposition 4.1 that $\pi$ extends continuously to $\bar{\Lambda}$.

Q.E.D.

\section{Lattices in higher RANK GROUPS AND CAT(-1)-SPACES}

In this section we prove Theorem 0.4. An ingredient in its proof is the determination of Furstenberg boundaries of $G(k)$, where $G$ is a reductive group defined over a local field $k$. These results (Proposition 5.1, Corollary 5.2) are well known when $k=\mathbb{R}, \mathbb{C}$ (see $[\mathrm{Fu}], \S 4)$; for ease of reference, we have included proofs valid for arbitrary local fields. Now we fix some notations used throughout this section.

For a connected reductive group $G$ defined over $k$, let $S$ denote a maximal $k$-split torus of $G,{ }_{k} W:=N(S) / Z(S)$ the Weyl group relative to $k, P$ a minimal parabolic $k$-subgroup containing $Z_{G}(S), P^{-}$the opposite parabolic $k$-subgroup containing $Z_{G}(S)$ and $U$ (resp. $U^{-}$) the unipotent radical of $P$ (resp. $P^{-}$). Let ${ }_{k} \Phi$ be the set of roots of $G$ w.r.t. $S$, which we assume given the ordering such that $P$ is associated to ${ }_{k} \Phi^{+}$, and let ${ }_{k} \Delta$ be the corresponding set of simple roots.

Proposition 5.1. Let $G$ be a connected reductive group defined over a local field $k$ and $Q<G$ a parabolic k-subgroup. The homogeneous space $G(k) / Q(k)$ is a Furstenberg boundary of $G(k)$.

Proof. We may assume $Q \supset P$. Let $\mu$ be a $G(k)$-quasi-invariant probability measure on $G(k) / P(k)$ and $\nu$ its direct image on $G(k) / Q(k)$ via the canonical projection map. It suffices to show that $\mu$ can be contracted to a Dirac mass. Indeed, this implies that $\nu$ can be contracted to a Dirac mass and, since supp $\nu=G(k) / Q(k)$, that every probability measure on $G(k) / Q(k)$ can be contracted to a Dirac mass (see [Az], Lemma II. 11).

Let

$$
G(k)=\bigsqcup_{w \in \epsilon_{k} W} U(k) w P(k)
$$


be the Bruhat decomposition of $G(k)$ and $\sigma \in{ }_{k} W$ the longest element. It follows from [Bo] (Corollary 21.28 and Theorem 21.26) that $U(k) \sigma P(k)$ is open and dense in $G(k)$. One concludes, using [Ber-Ze], 6.15, Theorem A, that the orbit map

$$
\begin{aligned}
U(k) & \longrightarrow G(k) / P(k) \\
u & \longmapsto u \sigma P(k)
\end{aligned}
$$

identifies $U(k)$ with an open dense subset $V$ of $G(k) / P(k)$.

Fix $t \in S(k)$ such that $|\alpha(t)|<1 \forall \alpha \in{ }_{k} \Delta$. For any eigenvalue $\lambda$ of $\left.A d(t)\right|_{\text {Lie } U}$, we have then $|\lambda|<1$. It follows then from the existence of an $S$-equivariant $k$-isomorphism of algebraic varieties $U \rightarrow \operatorname{Lie}(U)$ (foll. from [Bo-Ti], Theorem 4.15, and [Bo-Sp], Corollary 9.12, see [Ma], Proposition 1.3.3(i)) that $\operatorname{Int}(t)$ acts contracting on $U(k)$ and hence $\left.\lim _{n \rightarrow \infty} t_{*}^{n} \mu\right|_{V}=\mu(V) \cdot \delta_{\sigma P(k)}$.

It remains to show that the $\mu$-measure of the complement of $V$ is zero, or what amounts to the same, that the Haar measure of $U(k) w P(k)$ is zero for all $w \neq \sigma$.

Fix $w \in{ }_{k} W,{ }_{k} \Phi_{w}^{\prime}:=\left\{\alpha \in{ }_{k} \Phi^{+}: w^{-1}(\alpha)<0\right\},{ }_{k} \Phi_{w}=\left\{\alpha \in{ }_{k} \Phi^{+}: w^{-1}(\alpha)>0\right\}$ and let $U_{w}^{\prime}, U_{w}$ be the unipotent subgroups of $U$ associated resp. to ${ }_{k} \Phi_{w}^{\prime},{ }_{k} \Phi_{w}$. Then $U=U_{w}^{\prime} \cdot U_{w}$ and we have $w^{-1} U_{w}^{\prime} w \subset U^{-}, w^{-1} U_{w} w \subset U$ (see [Bo], 21.14). Set $U_{w}^{\prime \prime}:=w^{-1} U_{w}^{\prime} w$ so that $w^{-1} U(k) w P(k)=U_{w}^{\prime \prime}(k) \cdot P(k)$. The map

$$
\begin{aligned}
U^{-}(k) \times P(k) & \longrightarrow U^{-}(k) \cdot P(k) \subset G(k) \\
(\bar{u}, p) & \longmapsto \bar{u} \cdot p
\end{aligned}
$$

is a homeomorphism onto the open dense subset $U^{-}(k) \cdot P(k)$ and in these coordinates the restriction to $U^{-}(k) P(k)$ of the Haar measure of $G(k)$ is $d \bar{u} \times d p$ where $d \bar{u}$ is a left-invariant Haar measure on $U^{-}(k)$ and $d p$ a right invariant Haar measure on $P(k)$.

If now $U(k) w P(k)$ has positive Haar measure, then $U_{w}^{\prime \prime}(k) \cdot P(k)$ has positive $d \bar{u} \times d p$ measure and hence by Fubini's theorem, $U_{w}^{\prime \prime}(k)$ is a closed subgroup of $U^{-}(k)$ of positive $d \bar{u}$ measure. In particular, $U_{w}^{\prime \prime}(k)$ is open in $U^{-}(k)$ and so is $U_{w}^{\prime \prime}(k) P(k)$ in $U^{-}(k) P(k)$. The later set being open in $G(k)$, we deduce that $U(k) w P(k)$ is open in $G(k)$ and hence, since $U(k) \sigma P(k)$ is open dense, we must have $w=\sigma$. Q.E.D.

Corollary 5.2. Let $G$ be a connected reductive group defined over a local field $k, Q$ a parabolic $k$-subgroup and $\mu$ a $G(k)$-quasi-invariant probability measure on $G(k) / Q(k)$. The map $P: L^{\infty}(G(k) / Q(k), \mu) \rightarrow C(G(k))$, defined by $\operatorname{Pf}(g):=$ $\int_{G(k) / Q(k)} f(g x) d \mu(x)$, is injective.

Proof. Since $G(k) / Q(k)$ is a Furstenberg boundary, the map $P$ restricted to the subspace of continuous functions is injective.

Let $f \in L^{\infty}(\mu)$ with $P f=0$. For all $h \in G(k)$ and $F \in C_{00}(G(k))$ a continuous function of compact support, we have:

$$
\int_{G(k) / Q(k)} d \mu(x) \int_{G(k)} f(g h x) F(g) d g=\int_{G(k)} P f(g) F\left(g h^{-1}\right) d g=0 .
$$

Here $d g$ denotes a right-invariant Haar measure on $G(k)$. Since $G(k)$ acts transitively on $G(k) / Q(k)$, the function

$$
u_{F}(x):=\int_{G(k)} f(g x) F(g) d g
$$

is continuous and the above equality reads $P u_{F}=0$. Hence $u_{F}=0$ for all $F \in$ $C_{00}(G(k))$ and therefore $f=0$.

Q.E.D. 
Lemma 5.3. Let $G$ be a connected, simply connected, almost $k$-simple group and $H$ a locally compact second countable group. Any nontrivial continuous homomorphism $\pi: G(k) \rightarrow H$ is proper.

Proof. Assume that $\pi$ is not proper. In particular, $\operatorname{rank}_{k} G \geq 1$. Let $S^{\prime}:=\{t \in$ $\left.S(k):|\alpha(t)| \leq 1, \forall \alpha \in{ }_{k} \Delta\right\}$ and $M$ a compact subgroup such that $G(k)=$ $M S^{\prime} M$ (see [Ma], I. Theorem 2.2.1 and references therein). Since $\pi$ is not proper, there exists a sequence $\left(t_{n}\right)_{n \in \mathbb{N}}$ in $S^{\prime}$, leaving every compact subset of $S^{\prime}$, and such that $h:=\lim _{n \rightarrow \infty} \pi\left(t_{n}\right) \in H$ exists. Passing to a subsequence, we may assume $\lim _{n \rightarrow \infty} \alpha\left(t_{n}\right)=0$ for some $\alpha \in{ }_{k} \Delta$. Hence, there exists $u \in U, u \neq e$ with $\lim _{n \rightarrow \infty} t_{n} u t_{n}^{-1}=e$ and hence $e=\lim _{n \rightarrow \infty} \pi\left(t_{n} u t_{n}^{-1}\right)=h^{-1} \pi(u) h$. This implies that Ker $\pi$ is not in the center of $G(k)$ and therefore Ker $\pi \supset G(k)^{+}=G(k)$ (see [Ti 1]).

Q.E.D.

Lemma 5.4. Let $G$ be a connected $k$-almost simple group with $\operatorname{rank}_{k} G \geq 2, P$ a minimal parabolic $k$-subgroup and $Q_{1}, Q_{2}$ proper standard parabolic $k$-subgroups of $G$. Then $G(k) \neq Q_{1}(k) \cdot Q_{2}(k)$.

Proof. Assume $G(k)=Q_{1}(k) \cdot Q_{2}(k)$. Writing $g=q_{1} q_{2}=q_{2}^{\prime} \cdot q_{1}^{\prime}$ with $q_{i}, q_{i}^{\prime} \in Q_{i}(k)$, we have for the unipotent radical $R_{u} Q_{i}$ of $Q_{i}: g R_{u} Q_{1} g^{-1}=q_{2}^{\prime} R_{u} Q_{1} q_{2}^{\prime-1} \subset Q_{2}$, since $R_{u} Q_{1} \subset Q_{2}$; similarly $g R_{u} Q_{2} g^{-1} \subset Q_{1}$.

Hence $g\left[R_{u} Q_{1}(k) \cap R_{u} Q_{2}(k)\right] g^{-1} \subset Q_{1}(k) \cap Q_{2}(k)$ for all $g \in G(k)$. Let $V_{i} \subset{ }_{k} \Delta$ be the proper subset of simple roots corresponding to $Q_{i}$ and take $\alpha_{i} \in{ }_{k} \Delta \backslash V_{i}$. Since ${ }_{k} \Phi$ is irreducible there exists a root $\gamma \in{ }_{k} \Phi^{+}$containing $\alpha_{1}+\alpha_{2}$, in particular $\gamma \notin\left[V_{1}\right] \cup\left[V_{2}\right]$ and hence $U_{(\gamma)}(k) \subset R_{u} Q_{1}(k) \cap R_{u} Q_{2}(k)$ (see [Bo], 21.11, for the definition of $\left.U_{(\gamma)}\right)$. The normal subgroup of $G(k)$ generated by $U_{(\gamma)}(k)$ is therefore contained in $Q_{1}(k) \cap Q_{2}(k)$; in particular $G(k)^{+} \subset Q_{1}(k) \cap Q_{2}(k)$. This is a contradiction.

Q.E.D.

Proof of Theorem 0.4. For $\alpha, 1 \leq \alpha \leq n$, we fix a minimal $k_{\alpha}$-parabolic subgroup $P_{\alpha}$ of $G_{\alpha}$ and a maximal $k_{\alpha}$-split torus $S_{\alpha}$ in $P_{\alpha}$. In this proof a subgroup $Q<$ $G=\prod_{\alpha=1}^{n} G_{\alpha}\left(k_{\alpha}\right)$ is called standard parabolic if $Q=\prod_{\alpha=1}^{n} Q_{\alpha}\left(k_{\alpha}\right)$, where $Q_{\alpha}$ is a standard $k_{\alpha}$-parabolic subgroup of $G_{\alpha}$. Let $\mathcal{L}_{\pi(\Gamma)} \subset Y(\infty)$ denote the limit set of $\pi(\Gamma), Q$ be a standard parabolic subgroup of $G$, assume that there exists a $\Gamma$-equivariant measurable map $\varphi: G / Q \rightarrow \mathcal{L}_{\pi(\Gamma)}$ and let $R<G$ be a standard parabolic subgroup with $R \neq G$. Let $x_{Q}:=e Q \in G / Q$ and $\mu_{R}$ be an $R$-quasiinvariant probability measure supported on $R x_{Q} \subset G / Q$.

Claim 1. For almost every $g \in G$, the restriction of $\varphi$ to $g R x_{Q}$ coincides $g_{*} \mu_{R^{-}}$a.e. with a continuous map $\varphi_{g}$, and either

(a) $\varphi_{g}\left(g R x_{Q}\right)=\varphi\left(g x_{Q}\right)$ for a.e. $g \in G$, or

(b) $\varphi_{g}\left(g R x_{Q}\right)=\mathcal{L}_{\pi(\Gamma)}$ for a.e. $g \in G$.

Let $T_{\alpha} \subset S_{\alpha}$ be a $k_{\alpha}$-split torus such that $L_{\alpha}:=Z_{G_{\alpha}}\left(T_{\alpha}\right)$ is a $k_{\alpha}$-Levi subgroup of $R_{\alpha}$; set $T:=\prod_{\alpha=1}^{n} T_{\alpha}\left(k_{\alpha}\right)$ and $L:=\prod_{\alpha=1}^{n} L_{\alpha}\left(k_{\alpha}\right)$. Then $L_{\alpha} \cap Q_{\alpha}$ is a $k_{\alpha}$-parabolic subgroup of $L_{\alpha}$ ([Bo], 21.13 (i)), $L x_{Q}=R x_{Q}$ and $L / L \cap Q$ is a Furstenberg boundary of $L$ (see Proposition 5.1). Hence, since $L / L \cap Q$ is $L$-equivariantly homeomorphic to $L x_{Q}$ ([Ber-Ze], 6.15, Theorem A), $L x_{Q}$ is a Furstenberg boundary of $L$. Composing $\varphi$ with the measure class preserving map:

$$
\begin{aligned}
G \times(R / R \cap Q) & \longrightarrow G / Q \\
(g, r) & \longmapsto g r x_{Q}
\end{aligned}
$$


and applying Fubini's theorem, we deduce that for a.e. $g \in G$ the restriction of $\varphi$

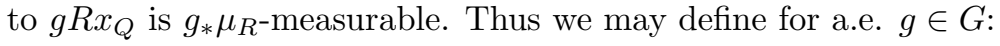

$$
\begin{aligned}
& \Phi(g)(\ell):=\varphi_{*}\left(g_{*} \ell_{*} \mu_{R}\right), \quad \ell \in L, \\
& F(g):=\operatorname{supp} \varphi_{*}\left(g_{*} \mu_{R}\right) .
\end{aligned}
$$

Since $\mu_{R}$ is $R$-quasi-invariant, (5.2) defines a $\Gamma$-equivariant measurable map

$$
F: G / R \longrightarrow K\left(\mathcal{L}_{\pi(\Gamma)}\right) \text {. }
$$

Since $\pi(\Gamma)$ is nonelementary, acts $c$-minimally on $Y$ and $\Gamma$ acts ergodically on $G / R \times G / R$, Proposition 3.1 implies that either

(a) $F(g)$ is a point for a.e. $g \in G$, or

(b) $F(g)=\mathcal{L}_{\pi(\Gamma)}$ for a.e. $g \in G$.

In the first case, the restriction of $\varphi$ to $g R x_{Q}$ is $g_{*} \mu_{R}$-a.e. constant for a.e. $g \in G$ and case (a) of the claim is proved.

Assume that the second case occurs. In particular we have $\Phi(g)(\ell) \in M_{3}^{1}(Y(\infty))$ for a.e. $g \in G$ and every $\ell \in L$. Since $L=Z_{G}(T)$ and $T \subset L \cap Q$, the subgroup $T$ acts trivially on $R x_{Q}=L x_{Q}$ and (5.1) defines a $\Gamma$-equivariant measurable map

$$
\Phi: G / T \longrightarrow F\left(L, M_{3}^{1}(Y(\infty))\right) .
$$

Since $R \neq G$, at least one of the tori $T_{\alpha}$ is of positive dimension and hence $T$ is noncompact. Since $\Gamma$ is an irreducible lattice it acts therefore ergodically on $G / T$; since the orbits of $\operatorname{Is}(Y)$ in $F\left(L, M_{3}^{1}(Y(\infty))\right.$ ) are locally closed (see Remark 4.2), we conclude that the essential image of $\Phi$ is in one $\operatorname{Is}(Y)$-orbit.

In particular, for a.e. $g \in G$, there is $\ell_{0} \in L$ such that for a.e. $\ell \in L$, there is $\tau_{g}(\ell) \in \operatorname{Is}(Y)$ with

$$
\Phi\left(g \ell_{0} \ell\right)=\tau_{g}(\ell) \Phi\left(g \ell_{0}\right) .
$$

Let $g \in G$ and $\ell, \ell_{0} \in L$ be such that (5.3) holds. For all $f \in C(Y(\infty))$ and all $\ell_{1} \in L$ we have therefore

$$
\int_{L x_{Q}} f\left(\varphi\left(g \ell_{0} \ell \ell_{1} x\right)\right) d \mu_{R}(x)=\int_{L x_{Q}} f\left(\tau_{g}(\ell) \varphi\left(g \ell_{0} \ell_{1} x\right)\right) d \mu_{L}(x) .
$$

Since $L x_{Q}$ is a Furstenberg boundary of $L$, Corollary 5.2 implies

$$
f\left(\varphi\left(g \ell_{0} \ell x\right)\right)=f\left(\tau_{g}(\ell) \varphi\left(g \ell_{0} x\right)\right)
$$

for all $f \in C(Y(\infty))$ and a.e. $x \in L x_{Q}$. Since $C(Y(\infty))$ is separable, we obtain

$$
\varphi\left(g \ell_{0} \ell x\right)=\tau_{g}(\ell) \varphi\left(g \ell_{0} x\right) \text { for a.e. } x \in L x_{Q} .
$$

In particular, since $F(g)=\mathcal{L}_{\pi(\Gamma)}$ and $\pi(\Gamma)$ acts $c$-minimally on $Y, \tau_{g}(\ell) \in \operatorname{Is}(Y)$ is uniquely determined by (5.4).

In this way we obtain for a.e. $g \in G$ a measurable map $\tau_{g}: L \rightarrow \operatorname{Is}(Y)$ which, in view of (5.4), is a measurable homomorphism. Therefore, $\tau_{g}$ coincides a.e. with a continuous homomorphism and hence, since $L$ acts transitively on $R x_{Q}$, the 
restriction of $\varphi$ to $g R x_{Q}$ coincides a.e. with a continuous map $\varphi_{g}$. In particular $F(g)=\varphi_{g}\left(g R x_{Q}\right)$ and hence $\varphi_{g}\left(g R x_{Q}\right)=\mathcal{L}_{\pi(\Gamma)}$. This proves Claim 1.

Now, since $G / P$ is a $(G, \Gamma)$-boundary and $\pi(\Gamma)$ is nonelementary, one obtains, using Corollary 3.2, the compactness and $\Gamma$-invariance of $\mathcal{L}_{\pi(\Gamma)}$, a $\Gamma$-equivariant measurable map

$$
\psi: G / P \longrightarrow \mathcal{L}_{\pi(\Gamma)}
$$

Let $\alpha:=\psi_{*}\left(\nu_{P}\right)$, where $\nu_{P}$ is a $G$-quasi-invariant probability measure on $G / P$. Since $\pi(\Gamma)$ acts minimally on $\mathcal{L}_{\pi(\Gamma)}$, we have $\operatorname{supp} \alpha=\mathcal{L}_{\pi(\Gamma)}$. According to Margulis' factor theorem ([Ma], IV Theorem 2.11) there exists $Q<G$, a standard parabolic subgroup, and

$$
\varphi: G / Q \longrightarrow \mathcal{L}_{\pi(\Gamma)},
$$

a bi-measurable bijection of the measure spaces $\left(G / Q, \nu_{Q}\right),\left(\mathcal{L}_{\pi(\Gamma)}, \alpha\right)$, such that $\varphi \circ \operatorname{pr}$ and $\psi$ coincide almost everywhere, where $\operatorname{pr}: G / P \longrightarrow G / Q$ is the canonical projection. Moreover $Q \neq G$ since $\pi(\Gamma)$ is nonelementary.

Claim 2. For any standard parabolic subgroup $R<G$ with $R \neq G$ we have either $R \subset Q$ or $R \cdot Q=G$.

We apply Claim 1 to $Q$ and $\varphi$. In case (a), the restriction of $\varphi$ to $g R x_{Q}$ is $g_{*} \mu_{R}$-almost everywhere constant for a.e. $g \in G$. Since $\varphi$ is injective, this implies that $g_{*} \mu_{R}$ is a Dirac mass for a.e. $g \in G$ and hence $R \subset Q$. In case (b), $\varphi\left(g R x_{Q}\right)$ has full $\alpha$-measure in $\mathcal{L}_{\pi(\Gamma)}$ for a.e. $g \in G$ and hence $R x_{Q}$ has full $\nu_{Q}$-measure in $G / Q$. The complement of $R x_{Q}$ in $G / Q$ being open, we must have $R x_{Q}=G / Q$ and hence $R \cdot Q=G$. This proves Claim 2 .

It follows from Claim 2 that $Q$ is a proper maximal standard parabolic subgroup of $G$. We may therefore assume $Q=Q_{1}\left(k_{1}\right) \times \prod_{\alpha=2}^{n} G_{\alpha}\left(k_{\alpha}\right)$, where $Q_{1}$ is a proper maximal standard $k_{1}$-parabolic subgroup of $G_{1}$. Assume that $\operatorname{rank}_{k_{1}} G_{1} \geq 2$ and take $R_{1}$ a proper standard $k_{1}$-parabolic subgroup of $G_{1}$ with $R_{1} \not \subset Q_{1}$. For $R:=$ $R_{1}\left(k_{1}\right) \times \prod_{\alpha=2}^{n} G_{\alpha}\left(k_{\alpha}\right)$ we have then $R \not \subset Q$ and (see Lemma 5.4) $R \cdot Q \neq G$, contradicting Claim 2 .

Hence $\operatorname{rank}_{k_{1}} G_{1}=1$ and, since $\sum_{k=1}^{n} \operatorname{rank}_{k_{\alpha}} G_{\alpha} \geq 2$, we must have $n \geq 2$. In particular $\Lambda:=\operatorname{pr}_{1}(\Gamma)$, where $\operatorname{pr}_{1}: G \longrightarrow G_{1}\left(k_{1}\right)$ denotes projection on the first factor, is a dense subgroup of $G_{1}\left(k_{1}\right)$.

Identifying $G / Q$ with $G_{1}\left(k_{1}\right) / Q_{1}\left(k_{1}\right)$, the $\Gamma$-equivariance of $\varphi: G_{1}\left(k_{1}\right) / Q_{1}\left(k_{1}\right)$ $\longrightarrow Y(\infty)$ reads: $\varphi\left(\operatorname{pr}_{1}(\gamma) x\right)=\pi(\gamma) \varphi(x)$ for a.e. $x \in G_{1}\left(k_{1}\right) / Q_{1}\left(k_{1}\right)$ and every $\gamma \in \Gamma$. Since the essential image of $\varphi$ is $\mathcal{L}_{\pi(\Gamma)}, \alpha\left(\operatorname{pr}_{1}(\gamma)\right):=\pi(\gamma)$ gives a well-defined homomorphism $\alpha: \Lambda \longrightarrow \operatorname{Is}(Y)$ with respect to which $\varphi$ is equivariant. Since $\Lambda$ is dense in $G_{1}\left(k_{1}\right)$ and $\alpha(\Lambda)=\pi(\Gamma)$ is nonelementary and acts $c$-minimally on $Y$, Proposition 4.1 implies that $\alpha$ extends continuously to $G_{1}\left(k_{1}\right)$ and hence is proper (Lemma 5.3).

Q.E.D.

Proof of Corollary 0.5. It suffices to show that $\overline{\pi(\Gamma)}$ is amenable. Indeed, since $\Gamma$ has property $(T)$ this implies that $\overline{\pi(\Gamma)}$ is compact and therefore fixes a point in $Y$. If $\overline{\pi(\Gamma)}$ is not amenable, Corollary 2.5(3) implies that $\overline{\pi(\Gamma)}$ is not elementary and hence (see 3.1) acts $c$-minimally on $\operatorname{Co}\left(\mathcal{L}_{\pi(\Gamma)}\right) \subset Y$, the closed convex hull of $\mathcal{L}_{\pi(\Gamma)}$. It follows then from Theorem 0.4 that there exists $\alpha$ with $\operatorname{rank}_{k_{\alpha}} G_{\alpha}=1$, which contradicts the assumptions.

Q.E.D. 


\section{Divergence Groups}

6.1. In this section we develop the part of Patterson-Sullivan theory for divergence groups acting on proper CAT(-1)-spaces needed in the proof of Theorem 0.2. As the proofs are almost the same as in the case of groups acting on hyperbolic spaces, we only indicate the modifications needed in order to treat the general case. The main results are stated in 6.3 .

6.2. Definitions. (a) Conical limit set: let $X$ be a proper CAT(-1)-space, $X(\infty)$ its ideal boundary, $G<\operatorname{Is}(X)$ a closed subgroup and $\pi: X \rightarrow G \backslash X$ the projection map. A point $\xi \in X(\infty)$ is a conical limit point of $G$, if for some (and hence any) geodesic ray $r:[0, \infty) \rightarrow X$ with $r(\infty)=\xi$, the map $\pi \circ r:[0, \infty) \rightarrow G \backslash X$ is not proper. The set $C_{G}$ of conical limit points of $G$ is a $G$-invariant Borel subset of $X(\infty)$.

(b) Geodesic flow: (compare $[\mathrm{Bou}], \S 2.8)$ the set

$$
\mathfrak{g} X:=\{c: \mathbb{R} \rightarrow X, c \text { is an isometry }\}
$$

endowed with the distance

$$
d_{\mathfrak{g}}\left(c_{1}, c_{2}\right):=\int_{-\infty}^{\infty} d\left(c_{1}(t), c_{2}(t)\right) \frac{e^{-|t|}}{2} d t
$$

is a proper metric space on which $\operatorname{Is}(X)$ acts by isometries. Observe that the subgroup of $\operatorname{Is}(X)$ consisting of the isometries acting trivially on $\mathfrak{g} X$ is compact. The geodesic flow is the $\mathbb{R}$-action on $\mathfrak{g} X$ defined by $g_{t} c(s):=c(s+t)$. It commutes with the action of $\operatorname{Is}(X)$. The fibers of the surjective $\operatorname{Is}(X)$-equivariant map

$$
\begin{aligned}
V: \mathfrak{g} X & \longrightarrow X(\infty) \times X(\infty) \backslash \operatorname{diag} \\
c & \longmapsto(c(-\infty), c(+\infty))
\end{aligned}
$$

are the $g_{t}$-orbits in $\mathfrak{g} X$. Each of these fibers carries therefore a canonical arc-length measure. Given a closed subgroup $G<\operatorname{Is}(X)$ and an $\alpha$-dimensional density $\mu$ for $G$, one verifies that the measure

$$
\frac{d \mu_{x}(\xi) \otimes d \mu_{x}(\eta)}{e^{-2 \alpha(\xi \cdot \eta)_{x}}} \text { on } X(\infty) \times X(\infty),
$$

where $(\xi \cdot \eta)_{x}$ denotes Gromov's scalar product, is independent of $x$ and hence invariant under the diagonal $G$-action. Combined with arc length measure on the fibers of the map $V$, one gets a $G$-invariant measure $\widetilde{m}_{\mu}$ on $\mathfrak{g} X$ which is also geodesic flow invariant. At this point we recall:

Lemma 6.1. Let $Y$ be a proper metric space, $G<\mathrm{Is}(Y)$ a closed unimodular subgroup and $\widetilde{m}$ a $G$-invariant Radon measure on $Y$. Given a Haar measure $d g$ on $G$, there is a unique Radon measure $m$ on $G \backslash Y$ such that

$$
\int_{G \backslash Y} d m(y) \int_{G} \psi(g y) d g=\int_{Y} d \widetilde{m}(y) \psi(y), \quad \forall \psi \in L^{1}(Y, \widetilde{m}) .
$$

We denote by $m_{\mu}$ the geodesic flow invariant measure on $G \backslash \mathfrak{g} X$ associated to $\widetilde{m}_{\mu}$ using the above lemma. 


\subsection{Statements.}

Theorem 6.2 (Hopf dichotomy). Let $G<\operatorname{Is}(X)$ be a nonelementary closed unimodular subgroup and $\nu$ an $\alpha$-dimensional density for $G$. Then the action of the geodesic flow on $\left(G \backslash \mathfrak{g} X, m_{\nu}\right)$ is either completely dissipative or completely recurrent. In the latter case, $\alpha=\delta_{G}$ and $\nu$ is, up to scaling, the unique $\delta_{G}$-dimensional density of $G$.

We recall that a continuous measure preserving action of $\mathbb{R}$ on a locally compact measure space $(Z, m)$ is

(a) completely recurrent if, for almost every $z \in Z$, the map

$$
\begin{aligned}
{[0, \infty) } & \longrightarrow Z \\
t & \longmapsto g_{t} z
\end{aligned}
$$

is not proper;

(b) conservative if there exists a positive $L^{1}$-function $\lambda: Z \rightarrow(0, \infty)$ such that $\int_{0}^{\infty} \lambda\left(g_{t} z\right) d t=+\infty$ for almost every $z \in Z$

(c) completely dissipative if, for almost every $z \in Z$, the map

$$
\begin{aligned}
{[0, \infty) } & \longrightarrow Z \\
t & \longmapsto g_{t} z
\end{aligned}
$$

is proper.

Definition. A divergence group is a nonelementary closed unimodular subgroup $G<\operatorname{Is}(X)$ of finite critical exponent, for which there exists a $\delta_{G}$-dimensional density $\mu$ such that the geodesic flow on $\left(G \backslash \mathfrak{g} X, m_{\mu}\right)$ is completely recurrent. For a divergence group $G$ we call Patterson-Sullivan density the (essentially) unique $\delta_{G}$-dimensional density of $G$ (see Theorem 6.2).

Theorem 6.3. Let $G<\operatorname{Is}(X)$ be a nonelementary closed unimodular subgroup and $\nu$ an $\alpha$-dimensional density. The following properties of the action of the geodesic flow on $\left(G \backslash \mathfrak{g} X, m_{\mu}\right)$ are equivalent:

(1) it is completely recurrent;

(2) it is conservative;

(3) it is ergodic.

For completeness we state the following result without proof. In the case of hyperbolic spaces it is due to Sullivan (see [Ni], Theorem 8.2.3, and [Su]); its proof may be adapted to the general case.

Theorem 6.4. For a closed unimodular nonelementary subgroup $G<\operatorname{Is}(X)$ the following properties are equivalent:

(1) $G$ is a divergence group;

(2) $\delta_{G}<+\infty$ and $\int_{G} e^{-\delta_{G} d(x, g y)} d g=+\infty$ for some (and hence any) $x, y \in X$.

Corollary 6.5. (1) Let $G_{1}<G_{2}<\mathrm{Is}(X)$ be closed nonelementary subgroups such that $G_{2} / G_{1}$ carries a finite $G_{2}$-invariant measure. Then $G_{1}$ is a divergence group if and only if $G_{2}$ is a divergence group. In this case, $\delta_{G_{1}}=\delta_{G_{2}}$ and the PattersonSullivan densities of $G_{1}$ and $G_{2}$ coincide up to a scaling factor. 
(2) Assume that $\operatorname{Is}(X) \backslash X$ is compact. Then any nonelementary lattice $\Gamma<$ $\operatorname{Is}(X)$ is a divergence group.

(3) Let $\Gamma<\operatorname{Is}(X)$ be a discrete divergence group, $\mu$ its Patterson-Sullivan density and $\mathrm{Com} \Gamma$ the commensurator of $\Gamma$ in $\operatorname{Is}(X)$. There exists a continuous homomorphism

$$
\chi: \overline{\operatorname{Com} \Gamma} \longrightarrow \mathbb{R}_{+}^{*}
$$

such that $\mu_{c \cdot x}=\chi(c) c_{*} \mu_{x}, \forall c \in \overline{\operatorname{Com} \Gamma}, \forall x \in X$.

Proof of Corollary 6.5. (1) Let $G_{1}<G_{2}<\operatorname{Is}(X)$ be closed nonelementary subgroups such that $G_{2} / G_{1}$ carries a $G_{2}$-invariant measure and let $\pi: G_{1} \backslash \mathfrak{g} X \rightarrow$ $G_{2} \backslash \mathfrak{g} X$ be the projection map.

If $G_{2}$ is a divergence group and $\mu$ its Patterson-Sullivan density, the associated measure $\widetilde{m}_{\mu}$ on $\mathfrak{g} X$ induces measures $m_{\mu}^{\prime}$ on $G_{1} \backslash \mathfrak{g} X$ and $m_{\mu}$ on $G_{2} \backslash \mathfrak{g} X$ such that

$$
\int_{G_{2} \backslash \mathfrak{g} X} d m_{\mu}(c) \int_{G_{1} \backslash G_{2}} d \dot{g} \psi(g c)=\int_{G_{1} \backslash \mathfrak{g} X} d m_{\mu}^{\prime}(c) \psi(c)
$$

for all $\psi \in L^{1}\left(G_{1} \backslash \mathfrak{g} X, m_{\mu}^{\prime}\right)$. Now the action of the geodesic flow on $G_{2} \backslash \mathfrak{g} X$ is conservative (Theorem 6.3). If $\lambda$ is a function on $G_{2} \backslash \mathfrak{g} X$ as in the definition of conservativity, it follows from $(*)$ that $\lambda \circ \pi \in L^{1}\left(G_{1} \backslash \mathfrak{g} X, m_{\mu}^{\prime}\right)$ and the geodesic flow is therefore conservative on $\left(G_{1} \backslash \mathfrak{g} X, m_{\mu}^{\prime}\right)$. Hence $G_{1}$ is a divergence group. It follows from the uniqueness statement in Theorem 6.2 that $\delta_{G_{1}}=\delta_{G_{2}}$ and $\mu$ is also the Patterson-Sullivan density of $G_{1}$.

Assume that $G_{1}$ is a divergence group and $\mu$ its Patterson-Sullivan density. One verifies that

$$
x \longmapsto \int_{G_{2} / G_{1}} d \dot{g} g_{*}^{-1} \mu_{g x}
$$

is a $\delta_{G_{1}}$-dimensional density for $G_{2}>G_{1}$. Hence, by Theorem 6.2 ,

$$
\int_{G_{2} / G_{1}} d g g_{*}^{-1} \mu_{g x}=\lambda \cdot \mu_{x}, \quad \forall x \in X
$$

where $\lambda>0$ is some constant. Therefore $\mu$ is a $\delta_{G_{1}}$-dimensional density of $G_{2}$. The recurrence of $g_{t}$ on $\left(G_{1} \backslash \mathfrak{g} X, m_{\mu}\right)$ implies the recurrence of $g_{t}$ on $\left(G_{2} \backslash \mathfrak{g} X, m_{\mu}\right)$. Hence $G_{2}$ is divergence; this implies $\delta_{G_{1}}=\delta_{G_{2}}$ and that $\mu$ is the Patterson-Sullivan density of $G_{2}$.

(2) By Proposition 1.7, $\operatorname{Is}(X)$ has finite critical exponent $\delta$ and by Proposition 1.1 there exists a $\delta$-dimensional density $\mu$ for $\operatorname{Is}(X)$. Since $\operatorname{Is}(X) \backslash \mathfrak{g} X$ is compact, $m_{\mu}$ is finite and hence the geodesic flow is recurrent. Hence $\operatorname{Is}(X)$ is a divergence group and assertion (2) now follows from assertion (1).

(3) Let $\Gamma<\operatorname{Is}(X)$ be a discrete divergence group. Observe that, since $\Gamma$ is nonelementary, any subgroup $\Gamma^{\prime}$ of finite index is nonelementary as well and hence a divergence group by assertion (1). Let $\delta$ be the critical exponent of $\Gamma, \mu$ the Patterson-Sullivan density of $\Gamma$ and $c \in$ Com $\Gamma$. Then, $x \rightarrow \mu_{x}$ and $x \rightarrow c_{*}^{-1} \mu_{c x}$ are $\delta$-dimensional densities of $\Gamma \cap c^{-1} \Gamma c$ and hence (assertion (1)) there exists $\chi(c)>0$ such that $c_{*}^{-1} \mu_{c x}=\chi(c) \mu_{x}, \forall x \in X$. Clearly the map $c \longrightarrow \chi(c)$ is a homomorphism. 
Since $x \rightarrow \mu_{x}$ is continuous, the homomorphism $\chi:$ Com $\Gamma \rightarrow \mathbb{R}_{+}^{*}$ is continuous

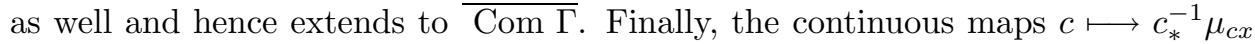
and $x \longrightarrow \chi(c) \mu_{x}$ defined on $\overline{C o m} \Gamma$ coincide on $\operatorname{Com} \Gamma$ and hence on $\overline{C o m} \Gamma$. Q.E.D

Proof of Theorem 0.2. (1) Let $\mu$ be the Patterson-Sullivan measure class on $X(\infty)$. Corollary 6.5(3) implies that $G$ preserves $\mu$ and Corollary 6.5(1) implies that the diagonal action on $(X(\infty) \times X(\infty), \mu \times \mu)$ of any finite index subgroup $\Gamma^{\prime}<\Gamma$ is ergodic. Corollary 1.4 and the uniqueness assertion in Theorem 6.2 imply then that $(X(\infty), \mu)$ is a weak $\Gamma$-boundary.

(2) follows from Corollary 3.2 and the fact that $(X(\infty), \mu)$ is a weak $\Gamma$-boundary. Q.E.D.

6.4. The rest of this section is devoted to the proof of Theorems 6.2 and 6.3.

For $x, y \in X$ and $R \geq 0, S(x, y, R) \subset X(\infty)$ denotes the set of endpoints of geodesic rays starting at $x$ and meeting $B(y, R)$. The next lemma follows from Sullivan's shadowing technique. For a proof, see [Ni], Theorem 4.3.2.

Lemma 6.6. Let $G<\operatorname{Is}(X)$ be a nonelementary closed subgroup, $\nu$ an $\alpha$-dimensional density of $G$ and $x \in X$. Then there are constants $R, c_{1}, c_{2}, k>0$ such that

$$
c_{1} \leq \frac{\nu_{x}(S(x, g x, R))}{e^{-\alpha d(x, g x)}} \leq c_{2},
$$

for all $g \in G$ with $d(x, g x) \geq k$.

In the above lemma, the hypothesis that $G$ is nonelementary is used to exclude the case where $\nu_{x}$ is a single atom. Observe that it follows from this lemma that no conical point is an atom of $\nu_{x}$.

Lemma 6.7. Let $G<\operatorname{Is}(X)$ be a nonelementary closed subgroup and $\nu$ an $\alpha$ dimensional density for $G$. Then $\delta_{G} \leq \alpha$.

Proof. Let $R, c_{1}, c_{2}, k>0$ be as in Lemma 6.6. For $N \in \mathbb{N}, N \geq k$ and $\epsilon>0$, Lemma 6.6 implies

$$
\begin{aligned}
& \int_{N \leq d(g x, x) \leq N+1} e^{-(\alpha+\epsilon) d(g x, x)} d g \\
& \quad \leq c_{1}^{-1} e^{-\epsilon N} \int_{N \leq d(g x, x) \leq N+1} \nu_{x}(S(x, g x, R)) d g \\
& \quad=c_{1}^{-1} e^{-\epsilon N} \int_{X(\infty)} d \nu_{x}(\xi) \int_{N \leq d(g x, x) \leq N+1} \chi_{g}(\xi) d g
\end{aligned}
$$

where $\chi_{g}$ denotes the characteristic function of $S(x, g x, R)$. Now observe that there is a constant $c>0$ such that, for all $g, h \in G$ with $|d(g x, x)-d(h x, x)| \leq 1$ and $S(x, g x, R) \cap S(x, h x, R) \neq \emptyset$, we have $d(g x, h x) \leq c$. Hence $(*)$ is bounded by $e^{-\epsilon N} c_{1}^{-1} \cdot \nu_{x}(X(\infty)) \cdot \int_{K} 1 d g$, where $K=\{k \in G d(k x, x) \leq c\}$. Summing over $N \in \mathbb{N}$, we get $\alpha+\epsilon \geq \delta_{G}, \forall \epsilon>0$ and the lemma is proved.

Q.E.D.

Corollary 6.8. Let $\Gamma<\operatorname{Is}(X)$ be a discrete subgroup and $\Gamma_{n}<\Gamma, n \in \mathbb{N}$, a family of nonelementary subgroups such that $\Gamma_{n} \subset \Gamma_{n+1}$, and $\bigcup_{n \in \mathbb{N}} \Gamma_{n}=\Gamma$. Then

$$
\delta_{\Gamma}=\lim _{n \rightarrow \infty} \delta_{\Gamma_{n}} .
$$


Proof. We may assume that the sequence $\left(\delta_{\Gamma_{n}}\right)_{n \in \mathbb{N}}$ is bounded. Fix $x \in X$ and choose for every $n$ a $\delta_{\Gamma_{n}}$-dimensional density $\mu_{n}$ for $\Gamma_{n}$, such that $\mu_{n, x}$ is probability. Let $\alpha \leq \delta_{\Gamma}$ be the limit of the increasing sequence $\left(\delta_{\Gamma_{n}}\right)_{n \in \mathbb{N}}$ (see Lemma 1.5) and $\mu \in M^{1}(X(\infty))$ an accumulation point of the sequence $\left(\mu_{n, x}\right)_{n \in \mathbb{N}}$. Since $\Gamma$ is the increasing union of $\left(\Gamma_{n}\right)_{n \in \mathbb{N}}$, we have $d\left(\gamma_{*} \mu\right)(\xi)=e^{-\alpha B_{\xi}(\gamma x, x)} d \mu(\xi), \forall \gamma \in \Gamma$. The formula $d \mu_{y}(\xi)=e^{-\alpha B_{\xi}(y, x)} d \mu(\xi)$ defines an $\alpha$-dimensional density for $\Gamma$ and hence $\alpha \geq \delta_{G}$ by Lemma 6.7 .

Q.E.D.

Lemma 6.9. Let $G<\operatorname{Is}(X)$ be a nonelementary closed subgroup, $\nu$ an $\alpha$-dimensional density for $G, C_{G}$ the set of conical points of $G$ and $A \subset C_{G}$ a $G$-invariant measurable subset. Then, either $\nu_{x}(A)=0$ or $\nu_{x}(A)=\nu_{x}(X(\infty))$.

For a proof see [Ni], Theorem 4.4.4.

Lemma 6.10. Let $G<\operatorname{Is}(X)$ be a nonelementary closed subgroup and $\nu$ an $\alpha$ dimensional density for $G$ with $\nu_{x}\left(C_{G}\right)>0$. Then

$$
\int_{G} e^{-\alpha d(g x, x)} d g=+\infty
$$

in particular $\alpha \leq \delta_{G}$.

Proof. Assume that $\int_{G} e^{-\alpha d(g x, x)} d g<+\infty$. Let $\epsilon>0$ and $K \subset G$ compact such that

$$
\int_{G \backslash K} e^{-\alpha d(g x, x)} d g<\epsilon .
$$

Let $K_{n} \subset K_{n+1}, n \in \mathbb{N}$, be a sequence of compact subsets of $G$ such that $G=$ $\bigcup_{n \in \mathbb{N}} K_{n}$. Observe that

$$
C_{G}=\bigcup_{N \in \mathbb{N}} \bigcap_{n \in \mathbb{N}}\left(\bigcup_{g \notin K_{n}} S(x, g x, N)\right) .
$$

Choose $N \in \mathbb{N}$ such that

$$
\nu_{x}\left(\bigcap_{n \in \mathbb{N}} \bigcup_{g \notin K_{n}} S(x, g x, N)\right) \geq \frac{\nu_{x}\left(C_{G}\right)}{2} .
$$

We may take $K$ large enough such that by Lemma 6.6

$$
c_{2}^{-1} \nu_{x}(S(x, g x, N+1)) \leq e^{-\alpha d(g x, x)}, \quad \forall g \notin K .
$$

Hence

$$
\int_{X(\infty)} d \nu_{x}(\xi) \int_{G \backslash K} d g \chi_{S(x, g x, N+1)}(\xi)<c_{2} \cdot \epsilon
$$

Set $U:=\{h \in G: d(x, h x)<1\}$.

Observe that, for every $\xi \in \bigcap_{n \in \mathbb{N}}\left(\bigcup_{g \notin K_{n}} S(x, g x, N)\right)$, there is $g \notin K$ such that $g U \subset G \backslash K$ and $\xi \in S(x, g u x, N+1), \forall u \in U$. This implies $\int_{G \backslash K} \chi_{S(x, g x, N+1)}(\xi) d g$ $\geq \int_{U} d u$ and hence

$$
\int_{U} d u \cdot \nu_{x}\left(\bigcap_{n \in \mathbb{N}}\left(\bigcup_{g \notin K_{n}} S(x, g x, N)\right)\right)<c_{2} \cdot \epsilon
$$


which implies $\nu_{x}\left(C_{G}\right)=0$ and proves the lemma.

Q.E.D.

Proof of Theorem 6.2. If $m_{\nu}$ is not completely dissipative, then $\nu_{x}$ gives positive measure to the set $C_{G}$ of conical points and hence, by Lemma 6.9, gives full measure to $C_{G}$. This implies that the geodesic flow is completely recurrent on $\left(G \backslash \mathfrak{g} X, m_{\nu}\right)$. If this is the case, then $\nu$ gives full measure to $C_{G}$ and hence $\alpha=\delta_{G}$ by Lemmas 6.7 and 6.10 .

If $\mu$ is any $\delta_{G}$-conformal density, then $\mu+\nu$ gives positive mass to $C_{G}$ and hence, by Lemma 6.9 , full mass to $C_{G}$. Furthermore, by Lemma $6.9, \nu_{x}+\mu_{x}$ and $\nu_{x}$ are ergodic and hence coincide up to a constant: $\mu_{x}=c(x) \cdot \nu_{x}, \forall x \in X$. Since

$$
\frac{d \mu_{x}(\xi)}{d \mu_{y}(\xi)}=\frac{d \nu_{x}(\xi)}{d \nu_{y}(\xi)}
$$

the function $x \rightarrow c(x)$ is constant.

Q.E.D.

Proof of Theorem 6.3. We concentrate on the implication $(1) \Longrightarrow(2)$. Once a function $\lambda: G \backslash \mathfrak{g} X \rightarrow(0, \infty)$ with suitable properties has been constructed, the proof of the implication (2) $\Longrightarrow(3)$ is verbatim Hopf's argument (see [Ho1], [Ho2]).

Lemma 6.11. Let $Y$ be a proper metric space, $G<\operatorname{Is}(Y)$ a closed unimodular subgroup, $\widetilde{m}$ a $G$-invariant positive measure on $Y$ and $m$ the corresponding measure on $G \backslash Y$. For the critical exponents $\delta_{\widetilde{m}}, \delta_{m}$ of $\widetilde{m}, m$ respectively, we have

$$
\delta_{m} \leq \delta_{\widetilde{m}} .
$$

Proof. Let $d$ denote distance on $Y, d_{Q}$ quotient distance on $G \backslash Y, \pi: Y \rightarrow G \backslash Y$ the projection map and $B_{Q}(z, R)$ a ball of radius $R$ with respect to the metric $d_{Q}$. We may assume that $\delta_{\widetilde{m}}<+\infty$. Observe that, for $y \in Y$ fixed,

$$
\delta_{\widetilde{m}}=\limsup _{R \rightarrow \infty} \frac{\log \tilde{m}(B(y, R))}{R} .
$$

Fix a Haar measure $d g$ on $G$ and choose $T$ finite such that the set

$$
U:=\{g \in G: d(g y, y) \leq T\}
$$

has measure at least 1 . Observe that $\forall g \in U: g B(y, R) \subset B(y, R+T)$. If $F$ denotes the characteristic function of $B(y, R+T)$, we have then

$$
\int_{G} F(g \zeta) d g \geq 1, \quad \forall \zeta \in B(y, R) .
$$

Since $\pi(B(y, R))=B_{Q}(\pi(y), R)$, we get

$$
\begin{aligned}
m\left(B_{Q}(\pi(y), R)\right) & \leq \int_{G \backslash Y} d m(\zeta) \int_{G} F(g \zeta) d g \\
& =\int_{Y} d \widetilde{m}(\zeta) F(\zeta)=\widetilde{m}(B(y, R+T)) .
\end{aligned}
$$

Hence $\delta_{m} \leq \delta_{\widetilde{m}}$

Q.E.D

Now let $X$ be a proper CAT $(-1)$-space, $G<\operatorname{Is}(X)$ a closed subgroup, $\nu$ an $\alpha$-dimensional density for $G, \widetilde{m}_{\nu}, m_{\nu}$ the corresponding measures on $\mathfrak{g} X, G \backslash \mathfrak{g} X$ respectively and $d_{Q}$ the quotient distance on $G \backslash \mathfrak{g} X$. Fix $c_{0} \in G \backslash \mathfrak{g} X$ and define, for $\beta>2 \alpha, \lambda(c)=e^{-\beta d_{Q}\left(c_{0}, c\right)}$. 
Lemma 6.12. (1) $\delta_{\widetilde{m}_{\nu}} \leq 2 \alpha$ and $\lambda \in L^{1}\left(G \backslash \mathfrak{g} X, m_{\nu}\right)$.

(2) There is a constant $K>0$ such that, for all $c, c^{\prime} \in G \backslash \mathfrak{g} X$ with $d_{Q}\left(c, c^{\prime}\right) \leq 1$,

$$
\left|\frac{\lambda(c)}{\lambda\left(c^{\prime}\right)}-1\right| \leq K \cdot d_{Q}\left(c, c^{\prime}\right) \text {. }
$$

(3) Assume that the geodesic flow on $\left(G \backslash \mathfrak{g} X, m_{\nu}\right)$ is completely recurrent. Then

$$
\int_{0}^{\infty} \lambda\left(g_{t} c\right) d t=+\infty, \quad \forall c \in G \backslash \mathfrak{g} X
$$

Proof. (1) Recall that for all $c_{1}, c_{2} \in \mathfrak{g} X$

$$
d\left(c_{1}(0), c_{2}(0)\right) \leq d_{\mathfrak{g}}\left(c_{1}, c_{2}\right) \leq d\left(c_{1}(0), c_{2}(0)\right)+2
$$

(see [Bou], Proposition 2.8.1).

Now fix $c_{0} \in \mathfrak{g} X$ and $R \geq 0$. If $d_{\mathfrak{g}}\left(c_{0}, c\right) \leq R$, then $d\left(c_{0}(0), c(0)\right) \leq R$ and hence $(c(-\infty), c(+\infty))_{c_{0}(0)} \leq R+C$, where $C$ is some constant depending only on $X$. Hence

$$
\widetilde{m}_{\nu}\left(B\left(c_{0}, R\right)\right) \leq 2 R \cdot e^{2 \alpha(R+C)} \cdot \nu_{c_{0}(0)}(X(\infty))^{2},
$$

which implies $\delta_{\widetilde{m}_{\nu}} \leq 2 \alpha$. The second assertion in (1) follows then from Lemma 6.11 .

(2) follows from the fact that $d_{Q}$ is a distance.

(3) Let $c \in \mathfrak{g} X$ project on a recurrent point in $G \backslash \mathfrak{g} X$. It follows from (*) that, for $T>0$ fixed, if $g_{t} c \in B\left(c_{0}, R\right)$, then $g_{t+s} c \in B\left(c_{0}, R+T+2\right), \forall s \in[0, T]$. Since $c$ projects to a recurrent point, there exist $t_{n} \rightarrow+\infty$ and $h_{n} \in G$ such that

$$
g_{t_{n}} c \in h_{n} B\left(c_{0}, R\right)
$$

and hence $g_{t_{n}+s} c \in h_{n} B\left(c_{0}, R+T+2\right), \forall s \in[0, T]$.

Since $\lambda$ has a positive lower bound on every compact set in $G \backslash \mathfrak{g} X$, we deduce that

$$
\int_{0}^{\infty} \lambda\left(g_{t} c\right) d t=+\infty .
$$

Q.E.D.

\section{Divergence groups aCting on trees}

In this section, we apply the results of Section 6 to the case of groups acting on locally finite trees in order to obtain examples of divergence groups. Notations and notions pertaining to graph theory are taken from Serre [Se].

7.1. Let $\mathcal{T}=(X, Y)$ be a locally finite tree, where $X$ is the set of vertices and $Y$ the set of edges. On the geometric realization $T$ of $\mathcal{T}$ there is a unique proper $\operatorname{CAT}(-1)$ distance $d$ for which all geometric edges have length 1 and whose restriction to $X \subset$ $T$ coincides with the combinatorial distance. Every automorphism $g \in$ Aut $\mathcal{T}=$ Is $(X)$ extends canonically to an isometry $g_{\text {ext }} \in \operatorname{Is}(T)$ of $T$ and we have Aut $\mathcal{T}=$ $\operatorname{Is}(T)$ except when $\mathcal{T}$ is the 2-regular tree $\mathcal{T}_{2}$. Every path $\alpha: \mathbb{P a t h}_{n} \rightarrow \mathcal{T}$ without backtracking (geodesic) extends to a geodesic $\alpha_{\text {ext }}:[0, n] \rightarrow T$, and for $n=\infty$ this 
extension gives an Aut $\mathcal{T}$ equivariant identification between $\mathcal{T}(\infty)$, the set of ends of $\mathcal{T}$, and $T(\infty)$. For a group $G$ acting by automorphisms on $\mathcal{T}$, the restriction from $T$ to $X$ provides a bijection between $\alpha$-dimensional conformal densities $T \rightarrow$ $M^{+}(T(\infty))$ for $G$ and $\alpha$-dimensional conformal densities $X \rightarrow M^{+}(\mathcal{T}(\infty))$ for $G$. Let $\mathfrak{g} \mathcal{T}$ denote the space of geodesics $\alpha: \mathcal{T}_{2} \rightarrow \mathcal{T}$, with $\mathbb{Z}$-action defined by

$$
g(n) c(m):=c(n+m), m \in V\left(\mathcal{T}_{2}\right)=\mathbb{Z}, n \in \mathbb{Z} .
$$

The map $\mathfrak{g} \mathcal{T} \rightarrow \mathfrak{g} T, c \rightarrow c_{\text {ext }}$, gives then an Aut $\mathcal{T}$-equivariant identification between the $\mathbb{R}$-action constructed from the time one suspension of the $\mathbb{Z}$-action on $\mathfrak{g} \mathcal{T}$, and the $\mathbb{R}$-action given by the geodesic flow on $\mathfrak{g} T$. In particular, the geodesic flow on $G \backslash \mathfrak{g} T$ is the time one suspension of the $\mathbb{Z}$-action on $G \backslash \mathfrak{g} \mathcal{T}$, which we will also call the geodesic flow. If $\mu: X \rightarrow M^{+}(\mathcal{T}(\infty))$ is a conformal density for $G, \mu_{\text {ext }}$ its extension to $T$, and $m_{\mu}$, resp. $m_{\mu_{\text {ext }}}$, the corresponding measures on $G \backslash \mathfrak{g} \mathcal{T}$, resp. $G \backslash \mathfrak{g} T$, then complete recurrence (resp. ergodicity) of the $\mathbb{Z}$-action on $\left(G \backslash \mathfrak{g} \mathcal{T}, m_{\mu}\right)$ is equivalent to complete recurrence (resp. ergodicity) of the geodesic flow on $\left(G \backslash \mathfrak{g} T, m_{\mu_{\text {ext }}}\right)$. In particular, the results of Section 6 apply to the $\mathbb{Z}$-action on $\left(G \backslash \mathfrak{g} \mathcal{T}, m_{\mu}\right)$.

7.2. For a locally finite tree $\mathcal{T}=(X, Y)$, let $S(\mathcal{T}(\infty))$ denote the space of locally constant functions on $\mathcal{T}(\infty)$ and $S(\mathcal{T}(\infty))^{*}$ its dual space. For $\alpha \in \mathbb{C}$, an $\alpha$ dimensional distribution is a map

$$
\mu: X \rightarrow S(\mathcal{T}(\infty))^{*}
$$

which satisfies $\mu_{x}(\varphi)=\mu_{y}\left(\varphi e^{-\alpha B(x, y)}\right) \forall x, y \in X, \forall \varphi \in S(\mathcal{T}(\infty))$. Here $B(x, y)$ denotes the extension to $\mathcal{T}(\infty)$ of the function $X \rightarrow \mathbb{R}, z \rightarrow d(x, z)-d(y, z)$ (see 1.1). For $g \in$ Aut $\mathcal{T}$ and $\mu$ an $\alpha$-dimensional distribution,

$$
\left(g_{*} \mu\right)_{x}:=g^{-1} \mu_{g x}, x \in X,
$$

defines an action of Aut $\mathcal{T}$ on the space $\mathcal{D}_{\alpha}$ of $\alpha$-dimensional distributions. For $\alpha \in$ $\mathbb{R}$, this action preserves $\mathcal{D}_{\alpha}^{+}$, the real cone of positive $\alpha$-dimensional distributions. Given $H<$ Aut $\mathcal{T}$, a closed subgroup, we observe that the subset $\left(\mathcal{D}_{\alpha}^{+}\right)^{H}$ of $H$-fixed vectors in $\mathcal{D}_{\alpha}^{+}$coincides with the set of $\alpha$-dimensional densities for $H$.

We define the following linear maps:

(a) $Q: \mathcal{D}_{\alpha} \rightarrow \mathbb{C}(Y), Q \mu(y):=\mu_{o(y)}\left(\chi_{s(y)}\right)$, where $\chi_{s(y)}$ is the characteristic function of the set $s(y) \subset \mathcal{T}(\infty)$ consisting of all geodesic rays $r: \mathbb{P a t h}_{\infty} \rightarrow \mathcal{T}$ having $y$ as initial edge.

(b) $R: \mathbb{C}(Y) \rightarrow \mathbb{C}(Y), R F(y)=\sum F\left(y^{\prime}\right)$, where the sum is over all edges $y^{\prime}$ satisfying $o\left(y^{\prime}\right)=t(y)$ and $y^{\prime} \neq \bar{y}$.

The linear maps $R$ and $Q$ are Aut $\mathcal{T}$-equivariant and $Q$ takes its values in

$$
E_{\alpha}:=\left\{F \in \mathbb{C}(Y): R F=e^{\alpha} F\right\}
$$

sending $\mathcal{D}_{\alpha}^{+}$into $E_{\alpha}^{+}:=\left\{F \in E_{\alpha}, F(Y) \subset[0, \infty]\right\}$.

Proposition 7.1. $Q: \mathcal{D}_{\alpha} \rightarrow E_{\alpha}$ is an Aut $\mathcal{T}$-equivariant isomorphism of vector spaces. In particular, for any subgroup $G<$ Aut $\mathcal{T}, Q$ induces a bijection between the set of $\alpha$-dimensional densities for $G$ and the set of $G$-invariant vectors in $E_{\alpha}^{+}$.

Proof. To verify injectivity, let $\mu \in \mathcal{D}_{\alpha}, x \in X$ and $y \in Y$ with $d(o(y), x)+1=$ $d(t(y), x)$. Then we have $\mu_{x}\left(\chi_{s(y)}\right)=e^{-n \alpha} \mu_{o(y)}\left(\chi_{s(y)}\right)$, where $n=d(o(y), x)$. In 
particular, if $Q \mu=0$ we have $\mu_{x}=0 \forall x \in X$. To obtain an inverse to $Q$, define for $F \in E_{\alpha}$, and $x, y$ as above:

$$
\mu_{x}\left(\chi_{s(y)}\right)=e^{-n \alpha} F(y) .
$$

With these definitions one has $\mu \in \mathcal{D}_{\alpha}$ and $Q \mu=F$.

Q.E.D.

Now we formulate some consequences of Proposition 7.1 in the context of graphs of groups. Let $\mathbb{A}=(\mathcal{A}, A)$ be a locally compact graph of groups. By this we mean that $A=(V, E)$ is a locally finite connected graph, for every $v \in V$ and $e \in E$ the groups $\mathcal{A}_{v}$ and $\mathcal{A}_{e}$ are compact and the monomorphisms $\alpha_{e}: \mathcal{A}_{e} \rightarrow \mathcal{A}_{o(e)}$ are continuous with image $\alpha_{e}\left(\mathcal{A}_{e}\right)$ of finite index $i_{A}(e)$ in $\mathcal{A}_{o(e)}$. Fixing a basepoint $a_{o} \in V$, the universal covering $\mathcal{T}=\widetilde{\left(\mathbb{A}, a_{o}\right)}$ is a locally finite tree and the image $G$ of the homomorphism $\pi_{1}\left(\mathbb{A}, a_{o}\right) \rightarrow$ Aut $\mathcal{T}$ is a closed subgroup of Aut $\mathcal{T}$. Let $\pi: \mathcal{T} \rightarrow A$ denote the canonical projection and $G_{f}=\{g \in$ Aut $\mathcal{T}: \pi \circ g=\pi\}$ the associated full group [Ba].

By definition, $G$ and $G_{f}$ have the same orbits in $Y$, hence $E_{\alpha}^{G}=E_{\alpha}^{G_{f}}$ and (using Proposition 7.1) $\mathcal{D}_{\alpha}^{G}=\mathcal{D}_{\alpha}^{G_{f}}$. In particular, any $\alpha$-dimensional density for $G$ is an $\alpha$-dimensional density for $G_{f}$.

The Aut $\mathcal{T}$-equivariant operator $R: \mathbb{C}(Y) \rightarrow \mathbb{C}(Y)$ induces an operator $R$ : $\mathbb{C}(E) \rightarrow \mathbb{C}(E)$ whose matrix $r: E \times E \rightarrow \mathbb{N}$ is given by

$$
r_{e_{1}, e_{2}}= \begin{cases}i_{\mathcal{A}}\left(e_{2}\right) & \text { if } t\left(e_{1}\right)=o\left(e_{2}\right), e_{1} \neq \bar{e}_{2}, \\ i_{\mathcal{A}}\left(e_{2}\right)-1 & \text { if } e_{1}=\bar{e}_{2}, \\ 0 & \text { otherwise }\end{cases}
$$

and $Q$ induces therefore a bijection

$$
\left(\mathcal{D}_{\alpha}^{+}\right)^{G}=\left(\mathcal{D}_{\alpha}^{+}\right)^{G_{f}} \longrightarrow F_{\alpha}^{+}:=\left\{F \in \mathbb{R}(E), R F=e^{\alpha} F, \quad F \geq 0\right\} .
$$

It follows then from Lemma 6.7, that if $G_{f}$ is nonelementary, its critical exponent $\delta_{G_{f}}$ can be computed by the formula

$$
\delta_{G_{f}}=\min \left\{\alpha \geq 0: F_{\alpha}^{+} \neq 0\right\} .
$$

According to [Ba-Ku], $G$ is unimodular if and only if $G_{f}$ is unimodular if and only if the edge indexed graph $\left(A, i_{A}\right)$ is unimodular. The homogeneous space $G_{f} / G$ being compact, it follows from Corollary 2.5(1) that if $G_{f}$ is unimodular it is nonelementary if and only if $G$ is nonelementary. Using Corollary 6.5(1) and 7.1, this implies that $G_{f}$ is a divergence group if and only if $G$ is a divergence group. According to $[\mathrm{Ba}], \mathcal{T}$ and $\pi: \mathcal{T} \rightarrow A$ only depend on the edge indexed graph $\left(A, i_{A}\right)$; in the sequel we will therefore call a locally finite edge indexed graph divergence if $G_{f}$ is a divergence group.

7.3. In this subsection we show that the $\mathbb{Z}$-action on $G_{f} \backslash \mathfrak{g} \mathcal{T}$ is isomorphic to a subshift and, that via this isomorphism, invariant measures coming from $\alpha$-dimensional densities of $G_{f}$ correspond to certain Markov measures associated to elements of $F_{\alpha}^{+}$. Concerning the $\mathbb{Z}$-action on $G \backslash \mathfrak{g} \mathcal{T}$ we mention that, even if $G$ is discrete and $G \backslash \mathcal{T}$ finite, this $\mathbb{Z}$-action need not be a finitely presented system. 
We will use the following basic constructions:

(a) To an edge indexed graph $\left(B, i_{B}\right)$ we associate the oriented line graph $\mathcal{L}^{+}\left(B, i_{B}\right)$ whose set of vertices is $E=E(B)$, the set of edges of $B$, and whose set of positive edges is

$$
\left\{\left(e_{1}, e_{2}\right) \in E \times E: t\left(e_{1}\right)=o\left(e_{2}\right) \text { and if } e_{2}=\overline{e_{1}} \text {, then } i_{B}\left(e_{2}\right)>1\right\} .
$$

(b) To an oriented graph $\mathfrak{a}^{+}$, we associate the shift space

$$
S_{\mathfrak{a}^{+}}=\left\{c: \mathcal{T}_{2}^{+} \rightarrow \mathfrak{a}^{+}, \text {morphism of oriented graphs }\right\}
$$

with $\mathbb{Z}$-action defined by

$$
g(n) c(i):=c(i+n), n \in \mathbb{Z}, i \in \mathbb{Z}, c \in S_{\mathfrak{a}^{+}} .
$$

Here $\mathcal{T}_{2}^{+}$is the 2-regular oriented tree, that is, its set of vertices is $\mathbb{Z}$ and its set of positive edges is $\{[i, i+1]: i \in \mathbb{Z}\}$. For $y_{1}, \ldots, y_{n}$, a sequence of consecutive vertices of a path in $\mathfrak{a}^{+}$, one defines the cylinder

$$
C\left(y_{1}, \ldots, y_{n}\right):=\left\{c: \mathcal{T}_{2}^{+} \rightarrow \mathfrak{a}^{+}: c(i)=y_{i}, 1 \leq i \leq n\right\} .
$$

The family of cylinders is a basis of open sets for a topology on $S_{\mathfrak{a}^{+}}$which is locally compact if $\mathfrak{a}^{+}$is locally finite. $G_{f}$.

In the sequel we use the following basic transitivity property of the full group

Lemma 7.2. Given $y_{1}, y_{2}$ edges of $\mathcal{T}$ satisfying $o\left(y_{1}\right)=o\left(y_{2}\right)$ and $\pi\left(y_{1}\right)=\pi\left(y_{2}\right)$, there exists $g \in G_{f}$ such that $g y_{1}=y_{2}$ and $g$ is the identity on the connected component of $\mathcal{T} \backslash\left\{y_{1}, y_{2}\right\}$ containing $o\left(y_{1}\right)$.

This lemma follows immediately from the description of $\left.\mathcal{T}=\widetilde{\left(\mathbb{A}, a_{0}\right.}\right)$ in terms of the edge indexed graph $\left(A, i_{A}\right)$ given in [Ba].

The coding of the $\mathbb{Z}$-action on $G_{f} \backslash \mathfrak{g} \mathcal{T}$ is now obtained as follows. The map $\mathfrak{g} \mathcal{T} \rightarrow S_{\mathcal{L}^{+}(\mathcal{T})}$, which to every geodesic $c: \mathcal{T}_{2} \rightarrow \mathcal{T}$ associates the morphism $c^{\prime}: \mathcal{T}_{2}^{+} \rightarrow \mathcal{L}^{+}(\mathcal{T})$ defined by $c^{\prime}(i):=c([i, i+1])$, is an Aut $\mathcal{T}$ and $\mathbb{Z}$-equivariant homeomorphism. The projection $\pi: \mathcal{T} \rightarrow A$ induces then a morphism $\pi^{+}$: $\mathcal{L}^{+}(\mathcal{T}) \rightarrow \mathcal{L}^{+}\left(A, i_{A}\right)$ and, using Lemma 7.2, a $\mathbb{Z}$-equivariant homeomorphism

$$
G_{f} \backslash \mathfrak{g} \mathcal{T} \longrightarrow S_{\mathcal{L}^{+}\left(A, i_{A}\right)} .
$$

We assume now that $G_{f}$ is unimodular and fix a Haar measure $m$ on $G_{f}$. For every path in $\mathcal{L}^{+}\left(A, i_{A}\right)$ with vertex sequence $y_{1}, \ldots, y_{n}$ choose $\tilde{y}_{1}, \ldots, \tilde{y}_{n}$ a lifted path in $\mathcal{L}^{+}(\mathcal{T})$ and let $K_{\tilde{y}_{1}, \ldots, \tilde{y}_{n}}$ be its stabilizer in $G_{f}$. This is an open compact subgroup of $G_{f}$ and, since $G_{f}$ is unimodular, its measure only depends on $y_{1}, \ldots, y_{n}$. Define

$$
m\left(y_{1}, \ldots, y_{n}\right):=m\left(K_{\tilde{y}_{1}, \ldots, \tilde{y}_{n}}\right) .
$$

We have, using Lemma 7.2:

$$
\frac{m\left(y_{1}, \ldots, y_{n}\right)}{m\left(y_{1}, \ldots, y_{n-1}\right)}=\frac{m\left(y_{n-1} y_{n}\right)}{m\left(y_{n-1}\right)}= \begin{cases}1 / i_{A}\left(y_{n}\right) & \text { if } y_{n-1} \neq \bar{y}_{n} \\ 1 / i_{A}\left(y_{n}\right)-1 & \text { if } y_{n-1}=\bar{y}_{n}\end{cases}
$$


To $\alpha \geq 0$ and $F \in F_{\alpha}^{+}$we associate the shift invariant measure

$$
m_{F} \in M^{+}\left(S_{\mathcal{L}^{+}\left(A, i_{A}\right)}\right)
$$

whose value on cylinders is given by

$$
\begin{aligned}
m_{F}(C(y)) & =\frac{F(\bar{y}) F(y)}{m(y)}, \\
m_{F}\left(C\left(y_{1}, \ldots, y_{n}\right)\right) & =\frac{F\left(\bar{y}_{1}\right) F\left(y_{n}\right) e^{-\alpha(n-1)}}{m\left(y_{1}, \ldots, y_{n}\right)}, n \geq 2 .
\end{aligned}
$$

Using the fact that $R F=e^{\alpha} F$ and $(*)$, one verifies that $m_{F}$ is Markov. Furthermore, for $\mu \in\left(\mathcal{D}_{\alpha}^{+}\right)^{G_{f}}$, the measures $m_{\mu} \in M^{+}\left(G_{f} \backslash \mathfrak{g} \mathcal{T}\right)$ and $m_{Q \mu} \in M^{+}\left(S_{\mathcal{L}^{+}\left(A, i_{A}\right)}\right)$ correspond to each other via the identification $G_{f} \backslash \mathfrak{g} \mathcal{T} \rightarrow S_{\mathcal{L}^{+}\left(A, i_{A}\right)}$. In particular, the total mass of $m_{\mu}$ equals

$$
\sum_{y \in E} \frac{F(\bar{y}) F(y)}{m(y)}, \text { where } F=Q \mu .
$$

7.4. We apply the preceding discussion to the edge indexed graph $\left(A, i_{A}\right)$ :

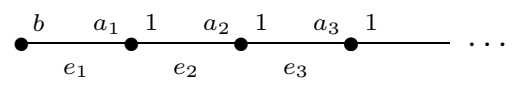

We assume that $b \geq 2$, not all $a_{i}$ 's are 1 and $P(X):=\sum_{k=1}^{\infty}\left(a_{k}-1\right) v_{k-1} X^{k}$ has positive radius of convergence $R$, where $v_{k}=a_{k} \cdot \ldots \cdot a_{1}$.

Proposition 7.3. (1) The critical exponent $\delta$ of $\left(A, i_{A}\right)$ is given by the formulas:

(a) if $P(R)>\frac{1}{b-1}$, then $P\left(e^{-2 \delta}\right)=\frac{1}{b-1}$,

(b) if $P(R) \leq \frac{1}{b-1}$, then $e^{-2 \delta}=R$.

(2) $\left(A, i_{A}\right)$ is divergent if and only if $P(R) \geq \frac{1}{b-1}$ in which case the PattersonSullivan measure is finite if and only if $P^{\prime}\left(e^{-2 \delta}\right)<+\infty$.

Proof. Fix $\alpha \in \mathbb{R}$. The equation $R F=e^{\alpha} F, F \in \mathbb{R}(E)$, is equivalent to

$$
\begin{gathered}
e^{\alpha} F\left(e_{i}\right)=F\left(e_{i+1}\right)+\left(a_{i}-1\right) F\left(\bar{e}_{i}\right), i \geq 1 ; \\
e^{\alpha} F\left(\bar{e}_{i}\right)=a_{i-1} F\left(\bar{e}_{i-1}\right), i \geq 2 ; \\
e^{\alpha} F\left(\bar{e}_{1}\right)=(b-1) F\left(e_{1}\right) .
\end{gathered}
$$

First we observe that $F\left(e_{1}\right)=0$ implies $F=0$. Hence we may assume $F\left(e_{1}\right)=1$. Equations (2) and (3) imply then:

$$
F\left(\bar{e}_{1}\right)=e^{-\alpha}(b-1) \text { and } F\left(\bar{e}_{j}\right)=e^{-j \alpha} v_{j-1}(b-1), j \geq 2 .
$$

Putting this into equation (1) we obtain

$$
e^{-j \alpha} F\left(e_{j+1}\right)=1-(b-1) \sum_{k=1}^{j} e^{-2 k \alpha}\left(a_{k}-1\right) v_{k-1} .
$$


We conclude that for every $\alpha \in \mathbb{R}$, there is a unique solution of $R F=e^{\alpha} F$ with $F\left(e_{1}\right)=1$ and, using (5), that this solution is in $F_{\alpha}^{+}$if and only if $P\left(e^{-2 \alpha}\right) \leq \frac{1}{b-1}$. Since $\delta=\min \left\{\alpha \geq 0: F_{\alpha}^{+} \neq(0)\right\}$ (see 7.2), we get assertion (1).

Let $F \in F_{\delta}^{+}$and $m_{F} \in M^{+}\left(S_{\mathcal{L}^{+}\left(A, i_{A}\right)}\right)$ be the associated shift invariant measure. Observe that $c \in S_{\mathcal{L}^{+}\left(A, i_{A}\right)}$ is not recurrent if and only if there exists $k \in \mathbb{Z}$ with $g(k) c \in \bigcap_{n \geq 1} C\left(e_{1}, \ldots, e_{n}\right)$. The definition of $m_{F}$ and equation (5) imply

$$
\begin{aligned}
m_{F}\left(\bigcap_{n \geq 1} c\left(e_{1}, \ldots, e_{n}\right)\right) & =\lim _{n \rightarrow \infty} F\left(\bar{e}_{1}\right) F\left(e_{n}\right) e^{-(n-1) \delta} \\
& =e^{-\delta}(b-1)\left[1-(b-1) P\left(e^{-2 \delta}\right)\right] .
\end{aligned}
$$

The measure of the set of nonrecurrent points is therefore 0 if and only if $P(R) \geq$ $\frac{1}{b-1}$ (see assertion (1)). The first assertion in (2) follows then from Theorem 6.3. For the second part, one uses the explicit formulas (4), (5) and the formula for the total mass of $m_{F}$ in 7.3 .

Q.E.D.

7.5. Cayley graphs and their fundamental group. Let $\mathcal{T}_{k}=(X, Y)$ be the $k$-regular tree, $k \geq 3, \Gamma<$ Aut $\mathcal{T}_{k}$ a subgroup acting freely without inversion on $\mathcal{T}_{k}, \mu$ an $\alpha$-dimensional density for $\Gamma$ and $\varphi_{\mu}(x):=\mu_{x}\left(\mathcal{T}_{k}(\infty)\right)$. Then $\varphi_{\mu} \in \mathbb{R}(X)$ is $\Gamma$-invariant and an eigenfunction of the operator $T F(x):=\sum_{o(y)=x} F(t(y))$, of eigenvalue $\lambda=(k-1) e^{-\alpha}+e^{\alpha}$. On the set of vertices $V$ of the quotient graph $\mathcal{C}:=\Gamma \backslash \mathcal{T}_{k}$, one defines a random walk with transition probabilities $P_{\mu}\left(x_{1}, x_{2}\right)=$ $\lambda^{-1} \varphi_{\mu}\left(x_{1}\right) / \varphi_{\mu}\left(x_{2}\right)$ if $x_{1}, x_{2} \in V$ are adjacent and $P_{\mu}\left(x_{1}, x_{2}\right)=0$ otherwise. Let $m_{\mu}$ be the geodesic flow invariant measure on $\Gamma \backslash \mathfrak{g} \mathcal{T}_{k}$ associated to $\mu$.

Theorem 7.4 [Co-Pa2]. If $\alpha \geq \frac{1}{2} \ell n(k-1)$, the random walk defined by $P_{\mu}$ is recurrent if and only if the geodesic flow on $\left(\Gamma \backslash \mathfrak{g} \mathcal{T}_{k}, m_{\mu}\right)$ is ergodic.

We apply this to $\mathcal{C}=\operatorname{Cay}(\Phi, S)$, the Cayley graph of a finitely generated group $\Phi$, with respect to a finite generating set $S$, satisfying $S=S^{-1}, e \notin S$ and $k:=$ $|S| \geq 3$. Let $\mu$ be the Patterson-Sullivan density for Aut $\mathcal{T}_{k}$, in particular we have $\alpha=\ln (k-1)$ and $\varphi_{\mu}(x)=1 \forall x \in X$. It follows from [Va] that $P_{\mu}$ is recurrent if and only if $\Phi$ is a finite extension of $(e), \mathbb{Z}$, or $\mathbb{Z}^{2}$, and in this case the above theorem implies that $\Gamma=\pi_{1}(\mathcal{C})$ is a divergence group. Conversely, assume that $\Gamma=\pi_{1}(\mathcal{C})$ is a divergence group with critical exponent $\delta$ and Patterson-Sullivan density $\nu$. Let $N$ be the fundamental group of the finite graph $\Phi \backslash \mathcal{C}$, in particular, $\Gamma$ is normal in $N$ and $N / \Gamma \cong \Phi$. Corollary 6.5(3) implies that there exists a homomorphism $\chi: N \rightarrow \mathbb{R}_{+}^{*}$ such that $\nu_{n_{*} x}=\chi(n) n_{*} \nu_{x}, \forall x \in V$ and $n \in N$. In particular, $\varphi_{\nu} \in \mathbb{R}(\Phi)$ verifies $\varphi_{\nu}(n \gamma)=\chi(n) \varphi_{\nu}(\gamma)$ and the equation $T \varphi_{\nu}(e)=\lambda \varphi_{\nu}(e)$ implies that

$$
\sum_{s \in S} \chi(s)=\lambda=(k-1) e^{-\delta}+e^{\delta} .
$$

We have $(k-1) e^{-\delta}+e^{\delta} \leq k$ for $\delta \in[0, \ln (k-1)]$, hence $\sum_{s \in S} \chi(s) \leq k$. Since $S=S^{-1}$, this implies $\chi=1$ and hence $\delta=0$ or $\delta=\ln (k-1)$. The condition $k \geq 3$ implies that $\Gamma$ is free nonabelian and hence $\delta>0$ by Corollary 2.5, which shows $\delta=\ell n(k-1)$. Since $\chi \equiv 1, \nu$ is the Patterson-Sullivan density of $N$ and since $N \backslash$ Aut $\mathcal{T}_{k}$ is compact, Corollary 6.5(1) implies that $\nu$ equals the Patterson-Sullivan density $\mu$ of Aut $\mathcal{T}_{k}$. Theorem 7.4 implies then that $P_{\mu}$ is recurrent and therefore [Va] $\Phi$ is a finite extension of $e, \mathbb{Z}$ or $\mathbb{Z}^{2}$. We obtain therefore 
Corollary 7.5. Let $\mathcal{C}=\operatorname{Cay}(\Phi, S)$ be the Cayley graph of a finitely generated group $\Phi$ with respect to a finite generating set $S$ satisfying $S=S^{-1} \not \supset e$ and $|S| \geq 3$. Then $\Gamma=\pi_{1}(\mathcal{C})<$ Aut $\mathcal{T}_{|S|}$ is a divergence group if and only if $\Phi$ is a finite extension of $(e), \mathbb{Z}$ or $\mathbb{Z}^{2}$.

\section{EXAMPLES}

8.1. Let $\mathcal{T}$ be a locally finite tree and $\Gamma<\operatorname{Aut} \mathcal{T}$ a uniform lattice; when $|\mathcal{T}(\infty)|>$ $2, \Gamma$ is nonelementary and hence a divergence group, furthermore, by a result of Liu [Li] $\operatorname{Com}_{\mathrm{Aut} \mathcal{T}} \Gamma$ is dense in Aut $\mathcal{T}$. The latter result was shown earlier by Bass and Kulkarni $[\mathrm{Ba}-\mathrm{Ku}]$ for a regular tree $\mathcal{T}$.

8.2. Let $\Gamma_{d}<$ Aut $\mathcal{T}_{2 d}$ be the fundamental group of the Cayley graph of $\mathbb{Z}^{d}$ w.r.t. the standard generators. We know that $\Gamma_{d}$ is a divergence group precisely when $d=2$ (Corollary 7.5); $\Gamma_{d}$ is not a lattice in Aut $\mathcal{T}_{2 d}$.

Proposition 8.1. The commensurator of $\Gamma_{d}$ is dense in Aut $\mathcal{T}_{2 d}$.

This result is an immediate consequence of

Proposition 8.2. Let $\mathcal{T}$ be a locally finite tree and $\Gamma<\operatorname{Aut}(\mathcal{T})$ a subgroup acting freely on $\mathcal{T}$. Let $Z=\Gamma \backslash \mathcal{T}$ be the quotient graph, $\pi: \mathcal{T} \rightarrow Z$ the covering map. Assume that for every pair of edges $e_{1}, e_{2}$ in $\mathcal{T}$ having a common vertex, if there exists $g \in$ Aut $\mathcal{T}$ such that $g e_{1}=e_{2}$ and $g e_{2}=e_{1}$, then there exists $h \in$ Aut $Z$ such that $h \pi\left(e_{1}\right)=\pi\left(e_{2}\right)$ and $h \pi\left(e_{2}\right)=\pi\left(e_{1}\right)$. Then $\overline{\operatorname{Com}_{\mathrm{Aut} \mathcal{T}} \Gamma}$ contains the subgroup of Aut $\mathcal{T}$ generated by all the vertex stabilizers.

Proof. It suffices to show that given any pair of equivalent edges $e_{1}, e_{2}$ in $\mathcal{T}$ as in the proposition and a finite subtree $F \subset \mathcal{T}$ which contains the edges $e_{1}, e_{2}$ at its boundary, i.e., such that the vertices $t\left(e_{i}\right), i=1,2$, are terminal vertices of $F$, then there exists an element $c \in \operatorname{Com}_{\mathrm{Aut}} \mathcal{T} \Gamma$ such that its restriction to $F$ is $\left.c\right|_{F}=\tau$, where $\tau: F \rightarrow F$ satisfies $\left.\tau\right|_{F \backslash\left\{e_{1}, e_{2}\right\}}=\mathrm{id}, \tau\left(e_{1}\right)=e_{2}$ and $\tau\left(e_{2}\right)=e_{1}$.

One obtains such an element $c$ by constructing an appropriate finite graph $W$ together with two covering maps $\varphi_{i}: W \rightarrow Z$ so that if we let $\pi_{W}: \mathcal{T} \rightarrow W$ be a covering map satisfying $\pi=\varphi_{1} \circ \pi_{W}$, then $\varphi_{2} \circ \pi_{W}=\varphi_{1} \circ \pi_{W} \circ \tau$. Denote by $f_{i}$ the edge $\pi\left(e_{i}\right)$. Let $W_{1}$ be the graph obtained by taking two copies $Z^{(1)}, Z^{(2)}$ of $Z$ and replacing the copies of the edges $f_{1}, f_{2}$ by edges going between the graphs $Z^{(1)}$ and $Z^{(2)}$. I.e., we omit $f_{i}^{(j)}, i=1,2, j=1,2$, and have new edges $\tilde{e}_{i}^{(j)}$ such that $o\left(\tilde{e}_{i}^{(1)}\right)=o\left(f_{i}^{(1)}\right), t\left(\tilde{e}_{i}^{(1)}\right)=t\left(f_{i}^{(2)}\right), o\left(\tilde{e}_{i}^{(2)}\right)=o\left(f_{i}^{(2)}\right)$, and $t\left(\tilde{e}_{i}^{(2)}\right)=t\left(f_{i}^{(1)}\right)$, $i=1,2$. Note that the graph $W_{1}$ may fail to be connected. In this case we will take $W_{1}$ to be the connected component containing the vertex $o\left(\tilde{e}_{i}^{(1)}\right)$. We continue the description as in the case where the original $W_{1}$ is connected, in the second case one should just ignore the parts referring to vertices and edges which happen to lie in the second connected component. Let $\varphi_{1}^{(1)}: W_{1} \rightarrow Z$ be the natural covering map and $h \in$ Aut $Z$ be the isometry interchanging $f_{1}$ and $f_{2}$. Using $h$ we define a covering map $\varphi_{2}^{(1)}: W_{1} \rightarrow Z$ as follows:

(a) Vertices and edges of $Z^{(1)}$ are mapped via the original identification between $Z^{(1)}$ and $Z$.

(b) Vertices and edges of $Z^{(2)}$ are mapped via composition of the original identification with the isometry $h$. 
(c) The edges $\tilde{e}_{i}^{(j)}$ are mapped by: $\tilde{e}_{1}^{(1)} \rightarrow f_{2}, \tilde{e}_{2}^{(1)} \rightarrow f_{1}, \tilde{e}_{1}^{(2)} \rightarrow f_{1}, \tilde{e}_{2}^{(2)} \rightarrow f_{2}$. The pair of finite covering maps $\varphi_{1}^{(1)}, \varphi_{2}^{(1)}: W_{1} \rightarrow Z$ induces an element $c_{1}$ in the commensurator of $\Gamma$ which interchange the edges $e_{1}$ and $e_{2}$ and fixes all other edges $e$ such that $o(e)=o\left(e_{i}\right)$. In order to obtain an element which stabilizes also the rest of the edges and vertices in the finite subtree $F$, we proceed by constructing new finite coverings $\varphi_{1}^{(k)}, \varphi_{2}^{(k)}: W_{k} \rightarrow Z, k=1,2, \ldots, N$, inducing elements of the commensurator interchanging $e_{1}$ and $e_{2}$ and each fixes a larger and larger part of $F$. Each graph $W_{k+1}$ will be constructed by adding a copy of $Z$ to $W_{k}$ and modifying certain pair edges. Assume that we already constructed $\varphi_{1}^{(k)}, \varphi_{2}^{(k)}: W_{k} \rightarrow Z$ such that the induced element $c_{k}$ of $\operatorname{Com}_{A u t} \mathcal{T} \Gamma$ interchanges $e_{1}$ and $e_{2}$ and fixes some part of the subtree $F$. Let $\pi_{W_{k}}: \mathcal{T} \rightarrow W_{k}$ be the covering map such that $\pi=\varphi_{1}^{(k)} \circ \pi_{W_{k}}$ and also $\pi_{W_{k}}\left(e_{i}\right)=\tilde{e}_{i}^{(1)}, i=1,2$. Consider the image of $F, \pi_{W_{k}}(F)$. If it is a tree, then the element $c_{k} \in \operatorname{Com}_{\mathrm{Aut} \mathcal{T}} \Gamma$ induced by the covering maps $\varphi_{1}^{(k)}, \varphi_{2}^{(k)}: W_{k} \rightarrow Z$ satisfies $\left.c_{k}\right|_{F}=\tau$ as required. Otherwise, there exist some loop in $\pi_{W_{k}}(F)$. Note that this loop contains also some edge lying inside one " $Z$ copy" in $W_{k}$ (this is easily checked for $k=1$ as well as for larger $k$ 's by induction), denote this edge by $f$. Let $Z^{\prime}$ be a new copy of $Z$ and let $f^{\prime} \in Z^{\prime}$ be the edge corresponding to $f$. Construct the graph $W_{k+1}$ by taking the union of $W_{k}$ with $Z^{\prime}$ and replacing the edges $f, f^{\prime}$ by the edges $\tilde{f}, \tilde{f}^{\prime}$ such that $o(\tilde{f})=o(f), t(\tilde{f})=t\left(\tilde{f}^{\prime}\right)$, $o\left(\tilde{f}^{\prime}\right)=o\left(f^{\prime}\right), t\left(\tilde{f}^{\prime}\right)=t(f)$. (Note that this new graph is still connected.) Next define the covering maps $\varphi_{i}^{(k+1)}: W_{k+1} \rightarrow Z, i=1,2$, in the obvious way. Consider the image $\pi_{W_{k+1}}(F)$. As either the number of loops decreased or some simple loop was replaced by a longer one, it follows that eventually we will reach some $N$ such that $\pi_{W_{N}}(F)$ will be a tree and the construction will be completed. Q.E.D.

8.3. Let $b \geq 4$ be an integer, $\mathcal{T}$ the universal covering and $\Gamma$ the fundamental group of the graph of groups

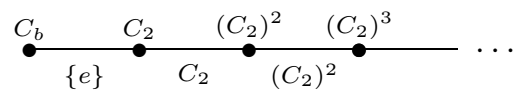

where $C_{m}$ denotes the cyclic group of order $m$, with monomorphisms $\left(C_{2}\right)^{m} \rightarrow$ $\left(C_{2}\right)^{m+1}=\left(C_{2}\right)^{m} \times C_{2}, x \mapsto(x, 0)$. Notice that the associated power series $P$ (see 7.4) is rational and hence $\Gamma<$ Aut $\mathcal{T}$ is a divergence group (Proposition 7.3(2)).

Theorem 8.3. $\Gamma<$ Aut $\mathcal{T}$ is a divergence group and its commensurator is dense in Aut $\mathcal{T}$. Moreover Aut $\mathcal{T}$ is not discrete.

We remark that if instead of the above graph of groups we consider the graph of groups having the same edge indexed graph but such that the groups $\left(C_{2}\right)^{m}$ are replaced by the cyclic groups $C_{2^{m}}$ then, as shown by Bass and Lubotzky in [Ba$\mathrm{Lu} 2$ ], the commensurator of the corresponding fundamental group is not dense. To see this they show that it has only finitely many subgroups of finite index and hence is essentially normalized by the commensurator. Since the subgroup of the automorphism group generated by stabilizers of vertices is simple (see [Ti2]), it follows that the commensurator cannot be dense.

The rest of this section is devoted to the proof of Theorem 8.3. Let $\mathcal{T}=(X, E)$ and $d(x)$ denote the degree of a vertex $x \in X$.

Set $X_{0}:=\{x \in X: d(x)=b\}$ and for every $x \in X$ define its level

$$
\ell(x):=\operatorname{dist}\left(x, X_{0}\right) \text {. }
$$


Let $X_{i}=\{x \in X: \ell(x)=i\}, X_{\leq i}=\{x \in X: \ell(x) \leq i\}$, and, for every $x \in X, E_{x}$ be the set of edges issued from $x$. For every $x \in X$ with $\ell(x) \geq 1, E_{x}=E_{x}^{+} \sqcup E_{x}^{-}$, where $E_{x}^{-}$is the pair of edges connecting $x$ to $X_{\ell(x)-1}$. A level increasing geodesic ray is a geodesic ray $r: \mathbb{N} \rightarrow X$ such that $\ell$ or is strictly increasing.

Labelling: for every $x$ with $\ell(x) \geq 1$ we label the edges $E_{x}^{-}$by $\{\alpha, \beta\}$, i.e. we fix a bijection $E_{x}^{-} \simeq\{\alpha, \beta\}$.

Numbering: for every $x \in X_{0}$, we number the edges $E_{x}$ by $0,1, \ldots, b-1$, i.e. we fix a bijection $E_{x} \simeq\{0,1, \ldots, b-1\}$. We arrange the labelling such that for every $x \in X_{0}$ the $b$ sequences of labels along the $b$ level-increasing geodesic rays starting at $x$ are the same. We arrange the numbering such that if we remove $X_{0}$ from $X$, then for every component $C$ of $X \backslash X_{0}$, all "leaf-edges" of $C$ get the same number.

Local data of an isometry: for $h \in \operatorname{Aut} \mathcal{T}$, the local data at $x \in X_{0}$ is the permutation of $\{0, \ldots, b-1\}$ it induces via the identifications of $E_{x}$ and $E_{h(x)}$ with $\{0, \ldots, b-1\}$. The local data at $x \in X \backslash X_{0}$ is the permutation induced by $h$ on $\{\alpha, \beta\}$ via the identifications of $E_{x}^{-}$and $E_{h(x)}^{-}$with $\{\alpha, \beta\}$. An isometry of $X$ is then completely determined by its local data at every point together with the image of one point. Of course, not every choice of local datas is realized by an isometry.

Let $\Gamma$ be the subgroup of Aut $\mathcal{T}$ consisting of all isometries $h$ satisfying:

$P_{1}$ : The local data of $h$ at level 0 vertices is a fixed power of the cycle $(0, \ldots$, $b-1)$.

$P_{2}$ : The local data of $h$ at $x \in X_{i}$ only depends on $i \geq 1$.

$P_{3}$ : For all $i$ 's except finitely many, the local data of $h$ at $X_{i}$ is trivial.

Let $r: \mathbb{N} \rightarrow X$ be a level increasing geodesic ray with $r(0) \in X_{0}$; set $x_{i}=r(i)$, $i \in \mathbb{N}$. To this ray we associate the following isometries of $X$ :

(a) $\rho \in$ Aut $\mathcal{T}$ defined by the conditions

(1) $\rho\left(x_{0}\right)=x_{0}$

(2) $\rho$ preserves all labels,

(3) the local data of $\rho$ at every $x \in X_{0}$ is the cycle $(0, \ldots, b-1)$.

(b) $i \geq 1, \tau_{i} \in \operatorname{Is}(X)$ defined by

(1) $\tau_{i}\left(x_{j}\right)=x_{j}, j \geq i$

(2) $\tau_{i}$ preserves the labels at all levels $j \neq i$;

(3) $\tau_{i}$ permutes the labels at all points of level $i$;

(4) $\tau_{i}$ preserves the numbering.

Using the properties of the labelling and numbering one verifies that $\rho, \tau_{i}, i \geq 1$, are well defined isometries. Let $Y(i)$ be the connected component of $X_{\leq i}$ containing $x_{0}$ and let

$$
\Gamma_{Y(i)}:=\{\gamma \in \Gamma: \gamma Y(i)=Y(i)\} .
$$

Lemma 8.4. (a) $\Gamma=\left\langle\rho, \tau_{i}: i \geq 1\right\rangle$ and $r(\mathbb{N})$ is a fundamental domain for the action of $\Gamma$ on $X$.

(b) $\Gamma_{Y(i)}=\left\langle\rho, \tau_{j}: 1 \leq j \leq i\right\rangle$.

This lemma is an easy verification which is left to the reader. Observe that part (a) of this lemma implies that $\Gamma$ is the fundamental group of the above graph of groups.

Let $\mathcal{L}_{i}$ be the set of connected components of $X_{\leq i}$ and define $Z_{1}, Z_{2} \in \mathcal{L}_{i}$ to be adjacent if the shortest geodesic connecting them intersects $X_{i}$ only in its endpoints. This puts on $\mathcal{L}_{i}$ the structure of a connected graph. Let $Z \in \mathcal{L}_{i}$ be adjacent to $Y(i)$ such that if $y \in Y(i)$ and $z \in Z$ denote the points realizing the distance $d(Y(i), Z)$ 
we have $y=x_{i}$. Then a suitable product of $\tau_{j}$ 's with $j \geq i+1$ defines an element of $\Gamma$ sending $Y(i)$ to $Z$ whose restriction $\tau_{Y(i), Z}$ to $Y(i)$ satisfies

(1) $\tau_{Y(i), Z}(y)=z$;

(2) $\tau_{Y(i), Z}$ has trivial local datas at all points of $Y(i)$.

In a similar way we get for every pair $Y, Z \in \mathcal{L}_{i}$ of adjacent components an isometry

$$
\tau_{Y, Z}: Y \longrightarrow Z
$$

verifying (1), (2) such that it is induced by an isometry $\widetilde{\tau}_{Y, Z}$ in $\Gamma$. In particular $\Gamma$ acts transitively on $\mathcal{L}_{i}$. We extend the definition of $\tau_{Y, Z}$ to any $Y, Z \in \mathcal{L}_{i}$ by

$$
\tau_{Y, Z}=\tau_{T_{n-1} T_{n}} \circ \ldots \circ \tau_{T_{1}, T_{2}} \circ \tau_{T_{0}, T_{1}}, \widetilde{\tau}_{Y, Z}=\widetilde{\tau}_{T_{n-1} T_{n}} \circ \ldots \circ \widetilde{T}_{T_{0} T_{1}}
$$

where $Y=T_{0} \equiv T_{1} \equiv \ldots \equiv T_{n}=Z$ is some geodesic joining $Y$ to $Z$ in $\mathcal{L}_{i}$. Notice that $\tau_{Y, Z}$ and $\widetilde{\tau}_{Y, Z}$ do not depend on the choice of this geodesic.

Let $G_{i}$ be the subgroup of Aut $\mathcal{T}$ consisting of all automorphisms $h$ such that

(1) $\forall Y, Z \in \mathcal{L}_{i}$ the diagram

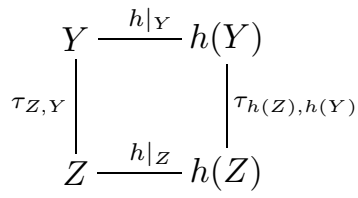

commutes.

(2) $\forall x \in X_{i}$, the local datas of $h$ on

$$
\left\{y \in X: \beta_{x}(y) \leq-1\right\}
$$

depend only on the level. Here $\beta_{x}$ is the Busemann function associated to the unique level increasing geodesic ray starting at $x$.

Proposition 8.5. (1) For every $Y, Y^{\prime} \in \mathcal{L}_{i}$ and every isometry $h: Y \rightarrow Y^{\prime}$, there is a unique extension of $h$ to an isometry $E(h) \in G_{i}$.

(2) $G_{i} \supset \Gamma$ and the restriction map $\operatorname{Stab}_{G_{i}}(Y) \longrightarrow \operatorname{Is}(Y), Y \in \mathcal{L}_{i}$, is an isomorphism.

(3) $\forall Y, Z \in \mathcal{L}_{i}: \widetilde{\tau}_{Y, Z}=E\left(\tau_{Y, Z}\right)$.

Proof. For $x \in X_{i}$ let $r_{x}$ denote the level increasing geodesic starting at $x$, and let $\beta_{x}$ be the associated Busemann function. For $N \in \mathbb{N}$ let $B(Y, N) \subset \mathcal{L}_{i}$ denote the ball of radius $N$ centered at $Y$ and $T_{N} \subset X$ denote the convex hull of

$$
\bigcup_{Z \in B(Y, N)} Z
$$

Assume that $E(h)$ is already defined on $T_{N}$ (this is so for $N=0$ ). Observe that the boundary $\partial T_{N}$ of $T_{N}$ consists of level $i$-vertices. Fix $x \in \partial T_{N}$. Then $E(h)$ extends in a unique way to $r_{x}(\mathbb{N})$, sending it to $r_{h(x)}(\mathbb{N})$. This fixes the local data of $E(h)$ at every vertex of $r_{x}(\mathbb{N})$ of level $\geq i+1$. Now $E(h)$ extends in a unique way to $\left\{y \in X: \beta_{x}(y) \leq 0\right\}$, sending this set to $\left\{y \in X: \beta_{h(x)}(y) \leq 0\right\}$ and such 
that the local data at every point of $\left\{y \in X: \beta_{x}(y) \leq-1\right\}$ only depends on its level.

Let $y \in X$ such that $\beta_{x}(y)=0, y \neq x$, and $Z \in S(Y, N+1)$ be the component with $y \in Z$. Let $Z^{\prime} \in S(h(Y), N+1)$ be the component with $h(y) \in Z^{\prime}$. Then there is a unique way of extending $E(h)$ to $Z$ such that the diagram

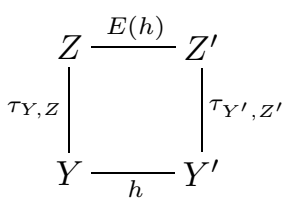

commutes.

This defines the extension of $E(h)$ to $T_{N+1}$. The construction shows that this extension of $h$ to an isometry in $G_{i}$ is unique. Assertion (2) follows from the definition of $\Gamma$ and the uniqueness statement in (1). Finally (3) follows from (2). Q.E.D.

Proposition 8.6. Let $Y \in \mathcal{L}_{i}$ and $\Gamma_{Y}^{\prime}:=\operatorname{Res}_{Y}\left(\Gamma_{Y}\right) \subset \operatorname{Is}(Y)$. Then we have $E\left(\operatorname{Com}_{\mathrm{Is}(Y)} \Gamma_{Y}^{\prime}\right) \subset \operatorname{Com}_{\mathrm{Aut} \mathcal{T}} \Gamma$.

Proof. Let $g \in \operatorname{Com}_{\mathrm{Is}(Y)} \Gamma_{Y}^{\prime}$ and choose representatives $\gamma_{1}, \ldots, \gamma_{r}$ of $\Gamma_{Y} / g^{-1} \Gamma_{Y}^{\prime} g \cap$ $\Gamma_{Y}^{\prime}$. We consider $\gamma_{i}$ as elements of $\Gamma_{Y}$. Given $\gamma \in \Gamma$ denote $\bar{\gamma}:=\left.\tau_{\gamma Y, Y} \circ \gamma\right|_{Y} \in \Gamma_{Y}^{\prime}$. Fix $j$ such that $\sigma:=g^{-1} \gamma_{j}^{-1} \bar{\gamma} g \in \Gamma_{Y}^{\prime}$. We have

$$
\begin{aligned}
\left.E(g)^{-1} \gamma_{j}^{-1} \gamma g\right|_{Y} & =\left.\left.\left.E(g)^{-1} \gamma_{j}^{-1}\right|_{\gamma Y} \circ \tau_{Y, \gamma Y} \gamma_{j} g g^{-.1} \gamma_{j}^{-1} \tau_{\gamma Y, Y} \gamma\right|_{Y} g\right|_{Y} \\
& =E\left(\gamma_{j} g\right)^{-1} \tau_{Y, \gamma Y} E\left(\gamma_{j} g\right) \sigma \\
& =\left.\tau_{Y, Z} \circ \sigma\right|_{Y} \text { where } Z=E\left(\gamma_{j} g\right)^{-1} \gamma Y
\end{aligned}
$$

We have $E\left(\tau_{Y, Z}\right) \in \Gamma, E(\sigma) \in \Gamma$ and

$$
\left.E(g)^{-1} \gamma_{j} \gamma E(g)\right|_{Y}=\left.E\left(\tau_{Y, Z}\right) E(\sigma)\right|_{Y},
$$

hence, by Proposition 8.5,

$$
E(g)^{-1} \gamma_{j} \gamma E(g)=E\left(\tau_{Y, Z}\right) E(\sigma) \in \Gamma .
$$

Q.E.D.

Corollary 8.7. $\operatorname{Com}_{\mathrm{Aut} \mathcal{T}} \Gamma$ is dense in Aut $\mathcal{T}$ and Aut $\mathcal{T}$ is not discrete.

Proof. Let $g \in$ Aut $\mathcal{T}, T \subset X$ a finite subtree and $i$ sufficiently big such that $T \cup g(T)$ is contained in a connected component $Y$ of $X_{\leq i}$. Since the quotient $\Gamma_{Y}^{\prime} \backslash Y$ is finite, there exists (see [Li]) $h \in \operatorname{Com}_{\text {Aut } \mathcal{T}} \Gamma_{Y}^{\prime}$ with $\left.h\right|_{T}=\left.g\right|_{T}$. According to Proposition 8.6, $E(h) \in \operatorname{Com}_{\mathrm{Aut} \mathcal{T}} \Gamma$ and $\left.E(h)\right|_{T}=\left.g\right|_{T}$. This proves the first assertion.

For the second assertion we observe that any connected component $Y$ of $X_{\leq 1}$ is isomorphic to the first barycentric subdivision of the $b$-regular tree $\mathcal{T}_{b}$. Via the homomorphism $E$, Aut $\mathcal{T}_{b}$ injects then into Aut $\mathcal{T}$. 


\section{REFERENCES}

[A'C-B] N. A'Campo and M. Burger, Réseaux arithmétiques et commensurateurs d'après G.A. Margulis, Invent. Math. 116 (1994), 1-25. CMP 94:05

[Ad1] S. Adams, Boundary amenability for word hyperbolic groups and an application to smooth dynamics of simple groups, Topology 33 (1994), 765-783. CMP 94:17

[Ad 2] - Reduction of cocycles with hyperbolic targets, preprint, 1993.

[Az] R. Azencott, Espaces de Poisson des groupes localement compacts, Lecture Notes in Math., vol. 148, Springer, Berlin and New York, 1970. MR 58:18748

[B] W. Ballmann, Lectures on spaces of nonpositive curvature, preprint, 1994.

[B-G-S] W. Ballmann, M. Gromov, and V. Schroeder, Manifolds of nonpositive curvature, Progr. in Math., vol. 61, Birkhäuser, Boston, 1985.

[B-Br] W. Ballmann and M. Brin, Polygonal complexes and combinatorial group theory, Geom. Dedicata 50 (1994), 165-191. MR 95e:57004

[Ba] H. Bass, Covering theory for graphs of groups, J. Pure Appl. Alg. 89 (1993), 3-47. MR 94j:20028

[Ba-Ku] H. Bass and R. Kulkarni, Uniform tree lattices, J. Amer. Math. Soc. 3 (1990), 843-902. MR 91k:20034

[Ba-Lu 1] H. Bass and A. Lubotzky, Rigidity of group actions on locally finite trees, Proc. London Math. Soc. 64 (1994), 541-575. CMP 94:16

[Ba-Lu 2] $\longrightarrow$, Tree lattices (In preparation).

[Be 1] N. Benakli, Polyèdres à géométrie locale donnée, C.R. Acad. Sci. Paris Sér. I 313 (1991), 561-564. MR 92k:52018

[Be 2] - Polyèdres hyperboliques à groupe d'automorphisme non discret, C.R. Acad. Sci. Paris Sér. I 313 (1991), 667-669. MR 93b:57005

[Ber-Ze] I.N. Bernstein and A.V. Zelevinski, Representations of the group $G L(n, F)$ where $F$ is a non-archimedean local field, Russian Math. Surveys 31 (1976), no. 3, 1-68. MR 54:12988

[Bo] A. Borel, Linear algebraic groups, Second enlarged edition, Springer, Berlin and New York, 1991. MR 92d:20001

[Bo-Sp] A. Borel and T.A. Springer, Rationality properties of linear algebraic groups II, Tôhoku Math. J. 20 (1968), 443-497. MR 39:5576

[Bo-Ti] A. Borel and J. Tits, Groupes réductifs, Inst. Hautes Études Sci. Publ. Math. 27 (1965), 55-150. MR 34:7527

[Bou] M. Bourdon, Actions quasi-convexes d'un groupe hyperbolique, flot géodésique, Thèse, Univ. de Paris-Sud, 1993.

[Bow] B.H. Bowditch, Some results on the geometry of convex hulls in manifolds of pinched negative curvature, Comment. Math. Helv. 69 (1994), 49-81. MR 94m:53044

[Bri-Ha] M. Bridson and A. Haefliger, Metric spaces of nonpositive curvature (In preparation).

[Co] M. Coornaert, Mesures de Patterson-Sullivan sur le bord d'un espace hyperbolique au sens de Gromov, Pacific J. Math. 159 (1993), 241-270. MR 94m:57075

[Co-Pa 1] M. Coornaert and A. Papadopoulos, Une dichotomie de Hopf pour les flots géodésiques associés aux groupes discrets d'isométries des arbres, Trans. Amer. Math. Soc. 343 (1994), 883-898. MR 94h:58131

[Co-Pa 2] Récurrence de marches aléatoires et ergodicité duflot géodésique surles graphes réguliers, Preprint IRMA, Strasbourg, 1994.

[Da] M.W. Davis, Nonpositive curvature and reflection groups, Lectures given at the eleventh annual western workshop in geometric topology, Park City, Utah, June 1994.

[Fu] H. Furstenberg, A Poisson formula for semisimple Lie groups, Ann. of Math. (2) 77 (1963), 335-383. MR 26:3820

[Gh-H] E. Ghys and P. de la Harpe (eds.), Sur les groupes hyperboliques d'après Gromov, Progr. Math., vol. 83, Birkhäuser, Boston, 1990.

[Gr] M. Gromov, Hyperbolic groups, Essays in Group Theory (S.M. Gersten, ed.), Math. Sci. Res. Inst. Publ., vol. 8, Springer, Berlin and New York, 1987. MR 89e:20070

[Hag] F. Haglund, Les polyèdres de Gromov, Thèse, Univ. de Lyon I, 1992.

[Ho1] E. Hopf, Statistik der geodätischen Linien in Mannigfaltigkeiten negativer Krümmung, Ber. Verh. Sächs. Akad. Wiss. Leipzig 91 (1933), 261-304. 
[Ho2] E. Hopf, Ergodic theory and the geodesic flow on surfaces of constant negative curvature, Bull. Amer. Math. Soc. 77 (1971), 863-877. MR 44:1789

[Ka] V. A. Kaimanovich, Ergodicity of harmonic invariant measures for the geodesic flow on hyperbolic spaces, J. Reine Angew. Math. 455 (1994), 57-103. CMP 95:01

[Li] Y. Liu, Density of the commensurability group of uniform tree lattices, J. Algebra 165 (1994), 346-359. MR 95c:20036

[L-M-Z] A. Lubotzky, S. Mozes, and R.J. Zimmer, Superrigidity of the commensurability group of tree lattices, Comment. Math. Helv. 69 (1994), 523-548. CMP 95:04

[Ma] G.A. Margulis, Discrete subgroups of semisimple groups, Ergeb. Math. Grenzgeb. (3)17, Springer, Berlin and New York, 1991. MR 92h:22021

[Mou] G. Moussong, Hyperbolic Coxeter groups, Ph.D. Thesis, Ohio State University, 1988.

[Ni] P.J. Nicholls, The ergodic theory of discrete groups, London Math. Soc. Lecture Notes, vol. 143, Cambridge Univ. Press, Cambridge and New York, 1989. MR 91i:58104

[Pa] S.J. Patterson, The limit set of a Fuchsian group, Acta Math. 136 (1976), 241-273. MR 56:8841

[Re] H. Reiter, Classical harmonic analysis and locally compact groups, Oxford Univ. Press, London and New York, 1968. MR 46:5933

[Se] J.-P. Serre, Trees, Springer, Berlin and New York, 1980. MR 82c:20083

[Su] D. Sullivan, Entropy, Hausdorff measures old and new, and limit sets of geometrically finite Kleinian groups, Acta Math. 153 (1984), 259-277. MR 86c:58093

[Ti1] J. Tits, Algebraic and abstract simple groups, Ann. of Math. (2) 80 (1964), 313-329. MR 29:2259

[Ti 2] Sur le groupe des automorphismes d'un arbre, Essays on Topology and Related Topics: Memoires dédiés à Georges de Rham (A. Haefliger and R. Narasimhan, eds.), Springer-Verlag, Berlin and New York, 1970, pp. 188-211. MR 45:8582

[Va] N.Th. Varapoulos, Analysis and geometry on groups, Proc. Internat. Congr. Math. (Kyoto 1990), 951-957.

[Zi 1] R. Zimmer, Curvature of leaves in amenable foliations, Amer. J. Math. 105 (1983), 1011-1022. MR 84k:58178

[Zi2] - Amenable ergodic group actions and applications to Poisson boundaries of random walks, J. Funct. Anal. 27 (1978), 350-372. MR 57:12775

[Zi3] _ Ergodic theory and semisimple groups, Monographs in Math., vol. 81, Birkhäuser, Boston, 1984. MR 86j:22014

ima, Université de Lausanne, Lausanne-Dorigny, Switzerland

Department of Mathematics, Hebrew University, Jerusalem, Israel 